\title{
Treillis et bases des groupes de Coxeter
}

\author{
Alain Lascoux $\left(^{*}\right)$ et Marcel-Paul Schützenberger \\ A Dominique Foata, \\ pionnier de la combinatoire \\ à qui elle doit tant
}

1. Introduction. - Le présent travail est une contribution à l'étude de l'ordre fort ( dit aussi de Bruhat) dans un groupe de Coxeter fini $W$.

Les définitions et propriétés relatives aux treillis, aux ensembles ordonnés, aux bases et aux treillis enveloppants, \&c. sont rassemblées de manière minimale dans la section 2 , les preuves et quelques exemples étant présentés en annexe, également de façon minimale.

La base $B$ d'un ensemble ordonné fini $(X, \leq)$ est le plus petit sousensemble tel que la comparaison de deux éléments pour l'ordre $\leq$ soit donné par l'ordre d'inclusion des projections (dans $2^{B}$ ) de ces éléments sur la base. La section 3 décrit les rapports entre les ordres faibles et fort sur les groupes de Coxeter, en les reliant à diverses algèbres de Hecke dégénérées. La base (pour l'ordre fort) est contenue dans l'ensemble de ce que nous appelons les bigrassmanniennes ( éléments $w \in W$ tels qu'il existe une seule paire de générateurs $\sigma, \sigma^{\prime}$ tels que $\left.\ell(\sigma \mu)<\ell(\mu), \ell\left(\mu \sigma^{\prime}\right)<\ell(\mu)\right)$.

La section 4 détaille le cas du groupe symétrique, pour lequel l'ordre fort a été décrit par Ehresmann en termes d'objets combinatoires que nous appelons clefs. La base est exactement l'ensemble des bigrassmanniennes. Ces dernières dépendent de 4 paramètres (en incluant l'ordre $n$ du groupe), et leur nombre est $\left(\begin{array}{c}n+1 \\ 3\end{array}\right)$. Nous montrons que le treillis enveloppant est distributif, en application d'un énoncé plus général sur les propriétés de clivage des treillis enveloppants.

Dans la section suivante, nous identifions les éléments du treillis enveloppant à des objets combinatoires très intéressants, les triangles, qui ont déjà été rencontrés dans un autre contexte, celui des matrices alternantes. Nous montrons que les permutations vexillaires se caractérisent de façon simple en terme de leur projection sur la base.

La section 6 utilise la même notion de clivage pour découper de façon itérée le groupe symétrique en intervalles remarquables, isomorphes aux intervalles inférieurs des bigrassmanniennes. Nous donnons les polynômes de Poincaré de ces intervalles.

La section 7 étend les résultats des sections 4 et 5 aux groupes de type $B_{n}$. Cette fois-ci, la base ne contient pas toutes les bigrassmanniennes,

(*) Soutenu par le PRC Math-Info et la bourse CEE n ${ }^{0}$ ERBCHRXCT930400 
mais le treillis enveloppant reste distributif. Le treillis de $B_{n}$ est en fait un sous-treillis du treillis enveloppant de $\mathfrak{S}(2 n)$.

Les bases de tous les groupes de Coxeter finis viennent d'être obtenues dans un article récent de M. Geck et S. Kim [G-Ki].

2. Treillis enveloppant. - Renvoyant les preuves à une annexe, nous nous limitons ici à énoncer les propriétés des treillis dont nous aurons besoin. La plupart de celles ci sont bien connues et nous faisons référence au grand traité de G.Birkhoff [Bi] ( et spécialement aux chapitres III et IX ) pour une théorie plus complète ainsi que pour les attributions de priorité.

Si $(X, \leq)$ est un ensemble ordonné et $x \in X$, nous notons $[x \leq]$ l'intervalle supérieur $\{y \in X: x \leq y\}$ et symétriquement, $[\leq x]:=\{y \in$ $X: y \leq x]\}$. L'infimum $\wedge Y=\operatorname{In} f(Y)$ d'une partie $Y$ de $X$ est l'unique élément $x \in X$ tel que

$$
[\leq x]=\cap\{[\leq y]: y \in Y\}
$$

s'il en existe un, et $\wedge Y=\emptyset$ sinon. Symétriquement, le supremum $\vee Y=$ $\operatorname{Sup}(Y)$ est l'unique $x^{\prime} \in X$ tel que

$$
\left[x^{\prime} \leq\right]=\cap\{[y \leq]: y \in Y\}
$$

s'il en existe un, $\emptyset$ sinon.

Un ensemble ordonné $(X, \leq)$ est un treillis (sous-entendu complet) ssi toute partie $Y$ non vide admet un Inf ( et donc ausi un Sup, ainsi qu'on le voit facilement, en prenant le Inf de l'ensemble des $z$ tels que $Y \subset[\leq z])$.

C'est un théorème classique dû à Mac Neille qu'il existe un treillis minimal unique $(T, \leq)$ et un isomorphisme d'ordre $X \rightarrow T$ commutant avec les opérations $\wedge$ et $\vee$ quand leur valeur est un élément de $X$.

Ce treillis $(T, \subset)$ est la plus petite famille de parties de $X$ qui contienne $X$, tous les intervalles supérieurs $[x \leq], x \in X$, et qui soit fermée par l'intersection ensembliste $\cap$. L'injection naturelle $x \longrightarrow[x \leq]$ de $(X, \leq)$ dans $(T, \subset)$ fait donc correspondre à l'opération $S u p$ de $(X, \leq)$ l'opération $\cap$ de $\left(2^{X}, \subset\right)$ qui est l'opération $\operatorname{Inf}$ de $(T, \subset)$; l'opération Inf de $(X, \leq)$ correspond quant à elle au $S u p$ de $(T, \subset)$, où cette dernière opération est définie de façon indirecte, ainsi que nous l'avons indiqué plus haut.

Nous appelerons $T$ le treillis enveloppant de $(X, \leq)$ bien que l'usage soit d'appeler $T$ la "complétion de $(X, \leq))$ ", parce que cette construction, qui ne fait appel à aucune hypothèse de finitude, constitue une généralisation 
naturelle de la construction de Dedekind (correspondant elle-même au cas où l'ordre sur $X$ est un ordre total).

On sait que de façon équivalente dans le cas fini, un treillis fini est un monoide commutatif idempotent finiment engendré (pour l'une quelconque des deux opérations), la relation d'ordre et la seconde opération étant alors canoniquement construites en terme de la première opération.

L'apport de la théorie des treillis au présent travail consiste essentiellement en les deux définitions suivantes et la proposition 2.5 :

Définition 2.1. - Une relation basique est un relation $\mathcal{R}$ entre deux ensembles $\bar{B}$ et $B$ telle que, en considérant $\mathcal{R} \in \bar{B} \times B$ comme une matrice booléenne, aucune ligne de $\mathcal{R}$ ne soit l'intersection de lignes, que la ligne composée entièrement de $\mathbf{1}$ n'appartienne pas à $\mathcal{R}$, et que les conditions symétriques soient satisfaites par les colonnes de $\mathcal{R}$.

Définition 2.2. - La base $B$ (resp. la cobase $\bar{B}$ ) de $(X, \leq)$ est l'ensemble des $x \in X \backslash \wedge X$ (resp. $x \in X \backslash \vee X$ ) tels que $x$ ne puisse être obtenu comme le $S u p$ (resp. le Inf) d'autres éléments, c'est-à-dire $x \in B$ (resp. $x \in \bar{B}$ ) si pour toute partie $Y$,

$$
x \notin Y \Rightarrow x \neq \operatorname{Sup}(Y)(\operatorname{resp} . x \neq \operatorname{In} f(Y)) .
$$

Nous supposerons désormais que tous les ensembles considérés sont finis.

Nous utiliserons la caractérisation suivante de la base, qui permet le calcul de cette dernière par simple inspection des triples d'éléments de $X$, puisque l'assertion que $x$ est minimal dans $X \backslash[\leq z]$ pour un $z \notin[x \leq]$ équivaut à $[<x] \backslash[\leq z]=\emptyset$.

Lemme 2.3. - Un élément $x \neq \wedge X$ appartient à la base $B$ ssi il est un élément minimal de $X \backslash[\leq z]$ pour au moins un $z \in X$.

Dans le cas particulier où $(X, \leq)$ est un treillis, on a que $x \in B$ ssi $x$ couvre un et un seul élément de $X$, c'est-à-dire ssi $x$ est un $\vee$-générateur.

La base satisfait de plus une propriété de minimalité :

Proposition 2.4. - La projection $\beta: X \ni x \rightarrow B \cap[\leq x] \in\left(2^{B}, \subset\right)$ est un isomorphisme d'ordre et l'on a $B \subset C$ pour toute partie $C$ de $X$ ayant la même propriété.

Ceci permet de coder chaque élément $x$ par l'ensemble des élements maximaux de $x \beta$, c'est à dire par ce que nous appelerons ses rectrices.

On a de même la projection $\bar{\beta}$ de chaque élément de $X$ sur la cobase.

Soit $\mathcal{R}^{\prime} \subset \bar{B} \times B$ la relation définie par $(\bar{b}, b) \in \mathcal{R}^{\prime}$ ssi $\bar{b} \geq b$, c'est-à-dire, de façon équivalente, ssi $b \in \bar{b} \beta$ ou $\bar{b} \in b \bar{\beta}$. 
Proposition 2.5. - La relation $\mathcal{R}^{\prime}$ est une relation basique.

Nous appelerons désormais la relation $\mathcal{R}^{\prime}$ précédente la relation basique de l'ensemble ordonné $(X, \leq)$.

ThÉorème 2.6. - Chaque ensemble ordonné a même relation basique que son treillis enveloppant.

Nous introduisons encore la

Définition 2.7. — La paire d'éléments $(\bar{x}, x)$ de l'ensemble ordonné $(X, \leq)$ clive $X$ ssi $X$ est l'union disjointe de $[\leq \bar{x}]$ et $[x \leq]$.

Les clivages de $X$ sont en bijection avec ceux de son treilis enveloppant, et les paires clivantes $(\bar{x}, x)$ sont nécessairement des éléments de $\bar{B} \times B$, ce qui permet d'énoncer un résultat classique de Birkhoff sous la forme modifiée suivante:

ThÉORÈme 2.8. - Une condition nécessaire et suffisante pour que le treillis enveloppant d'un ensemble ordonné $(X, \leq)$ soit distributif est qu'il existe un isomorphisme d'ordre bijectif entre sa base et sa cobase tel que chaque paire d'éléments associés donne un clivage.

Comme il est bien connu, il y a bijection, pour un treillis distributif, entre les antichaînes de la restriction de la relation d'ordre à la base et les éléments du treillis: c'est un cas particulier de la correspondance entre les éléments et leurs rectrices.

3. Ordres sur les groupes de Coxeter et algèbres de IwahoriHecke dégénérées. - Soit $(W, S)$ un groupe de Coxeter fini, $S$ un système de générateurs. L'algèbre d' Iwahori-Hecke $\mathcal{H}(W, p, q)$ est l'algèbre engendrée par des éléments $T_{s}$ vérifiant les relations de Coxeter, plus les relations de Hecke (homogénéisées)

$$
T_{s}^{2}=(p+q) T_{s}-p q
$$

où $p, q$ sont deux constantes commutant avec les $T_{s}$. Pour le groupe symétrique, nous nous sommes attachés à développer les cas $T_{s}^{2}=0$ (différences divisées), $T_{s}^{2}=T_{s}$ (différences divisées isobares), $T_{s}^{2}=-T_{s}$, sans oublier l'algèbre du groupe $\left(T_{s}^{2}=1\right)$, cf. [L-S 1], [L-S 2], et aussi [Ch] pour ce qui concerne les algèbres de Hecke affines. 
Employons des notations différentes pour distinguer les quatre cas spéciaux ci-dessus (pour les valeurs des paramètres $p, q$ respectivement égales à $(0,0),(0,1),(0,-1)$ et $(1,-1))$ :

$$
\partial_{s}, \pi_{s}, \bar{\pi}_{s}, s
$$

vérifiant respectivement

$$
\partial_{s}^{2}=0, \pi_{s}^{2}=\pi_{s}, \bar{\pi}_{s}^{2}=-\bar{\pi}_{s}, s^{2}=1 .
$$

Pour chacune de ces algèbres, nous considérerons aussi le monoide engendré par les générateurs $T_{i}$.

L'algèbre d' Iwahori-Hecke admet une base linéaire $\left\{T_{w}\right\}_{w \in W}$, en bijection avec les éléments du groupe, que nous noterons respectivement $\left\{\partial_{w}\right\},\left\{\pi_{w}\right\},\left\{\bar{\pi}_{w}\right\}$ et $\{w\}$ dans les cas spéciaux, l'algèbre étant elle-même $\mathcal{H}^{\partial}, \mathcal{H}^{\pi}, \mathcal{H}^{\bar{\pi}}$ et $\mathcal{H}^{s}$ respectivement.

Les $\partial_{w}$ permettent de définir la longueur des éléments de $W$, les décompositions réduites et l'ordre faible droit ou gauche sur $W$.

Définitions. - Etant donné $w \in W$, la longueur maximum d'une factorisation dans le monoide des $\partial_{i}: \partial_{w}=\partial^{1} \partial^{2} \cdots \partial^{k}$ (en facteurs $\neq 1$ ) est dite longueur de $w$ et notée $\ell(w)$. Une factorisation de longueur maximum est telle que les facteurs $\partial^{1}, \ldots, \partial^{k}$ sont des générateurs. La factorisation correspondante dans $W: w=s^{1} \cdots s^{k}$ est une décomposition réduite de $w$.

Deux éléments sont comparables pour l'ordre faible droit $: v \leq_{R} w$ ssi $\partial_{v}$ est facteur gauche de $\partial_{w}$, i.e. s'il existe $u \in W$ tel que

$$
\partial_{v} \partial_{u}=\partial_{w}
$$

Symétriquement, $v \leq_{L} w$ ssi $\partial_{v}$ est facteur droit de $\partial_{w}$.

Etant donnés deux éléments $w, w^{\prime}$ de $W$, il existe un plus petit $v \in W$ (pour l'ordre droit) tel que $\partial_{v}$ ait pour facteur gauche $\partial_{w}$ et $\partial_{w^{\prime}}$. En d'autres termes, l'intersection des idéaux $\partial_{w} \mathcal{H}^{\partial}$ et $\partial_{w^{\prime}} \mathcal{H}^{\partial}$ est un idéal principal $\left(=\partial_{v} \mathcal{H}^{\partial}\right)$.

Similairement, il existe un plus grand facteur gauche $\partial_{v}$ de $\partial_{w}$ et $\partial_{w^{\prime}}$.

L'existence d'un plus grand facteur gauche et d'un plus petit commun multiple signifie que $W$, muni de l'ordre $<_{R}$, est un treillis. Cette propriété a été montré par Guilbaud \& Rosensthiel [G-R] pour le groupe symétrique, et est due à Björner pour les autres groupes de Coxeter finis [Bj].

Symétriquement, l'ordre faible gauche donne une deuxième structure de treillis, image de la précédente par inversion des permutations.

A chacune des deux structures de treillis correspond une base.

Définitions. - Pour $w \in W$, l'ensemble des descentes (resp reculs) de $w$ est le sous-ensemble des générateurs de $W$ tels que $\partial_{w} \partial_{s}=0$ (resp. 
$\left.\partial_{s} \partial_{w}=0\right)$. Un élément est dit grassmannien s'il n'a qu'une descente. Une bigrassmannienne est un élément de $W$ ayant une seule descente et un seul recul.

La base de $W$, muni de l'ordre $<_{R}$, est constituée des éléments grassmanniens, puisque ceux-ci sont exactement les $w \in W$ ayant un et un seul successeur.

Soit $s$ un générateur du groupe de Coxeter $(W, \mathcal{S})$. Le sous-ensemble des éléments grassmanniens ayant $s$ pour descente, auquel on adjoint l'identité, est un treillis pour l'ordre $<_{L}(\mathrm{cf}$. $[\mathrm{B}-\mathrm{W}])$. Cet ensemble est usuellement noté $W^{s}$; ses éléments sont les représentants de longueur minimum des classes $W / W(\mathcal{S} \backslash s)$, où $W(\mathcal{S} \backslash s)$ est le parabolique engendré par tous les générateurs sauf $s$.

Le morphisme $p_{s}: W \rightarrow W^{s}$ qui à chaque élément de $W$ associe l'élément de longueur minimum de sa classe modulo $W(\mathcal{S} \backslash s)$ est défini par

$$
p_{s}(w)=g \Leftrightarrow \partial_{g} \text { est le plus grand facteur gauche de } w, g \in W^{s}
$$

Il est équivalent de rechercher les décompositions $\partial_{w}=\partial_{g} \partial_{v}, v \in$ $W(\mathcal{S} \backslash s)$, telles que $\partial_{v}$ soit de longueur maximale parmi les facteurs droits de $\partial_{w}$ appartenant à $W(\mathcal{S} \backslash s)$.

On peut aussi munir $W$ du plus petit ordre ( ordre bifaible) contenant $<_{L}$ et $<_{R}$ et étudier les propriétés des facteurs des $\partial_{w}$.

La considération des sous-mots conduit, quant à elle, à définir un ordre dit fort ou de Bruhat que nous noterons désormais $\leq$.

Définitions. - On a a $v \leq w$ ssi $\partial_{v}$ est un sous-mot de $\partial_{w}$, i.e. s'il existe deux factorisations

$$
\partial_{v}=\partial_{v_{1}} \partial_{v_{2}} \cdots \partial_{v_{k}} \quad \text { et } \quad \partial_{w}=\partial_{w^{\prime}} \partial_{v_{1}} \partial_{w^{\prime \prime}} \partial_{v_{2}} \partial_{w^{\prime \prime \prime}} \cdots \partial_{v_{k}} \partial_{w^{\prime \prime \prime \prime}}
$$

Il suffit en fait de ne considérer que les sous-mots d'une seule décomposition réduite arbitraire de $w$ pour obtenir tous les éléments $v \leq w$ (cf. [Bo]).

Les idéaux d'ordre pour l'ordre fort se décrivent aisément dans l'algèbre $\mathcal{H}^{\bar{\pi}}$. On a en effet la récurrence suivante $([\mathrm{Vr}])$ :

Lemme 3.1. - Soient $w \in W, s \in \mathcal{S}$, tels que $\ell(w)<\ell(w s)$. Alors l'intervalle inférieur $[\leq w]$ se décompose en deux sous-ensembles $\{v: v<w, v s<w\}$ et $\{u: u \leq w, u s \nless w\}$, et l'intervalle $[\leq w s]$ est égal $\grave{a}$

$$
[\leq w] \cup\{u s: u \leq w, u s \nless w\} \quad .
$$


Corollaire 3.2. - Pour tout w,

$$
\pi_{w}=\sum_{v \leq w} \bar{\pi}_{v}
$$

et

$$
\bar{\pi}_{w}=\sum_{v \leq w}(-1)^{\ell(w)-\ell(v)} \pi_{v}
$$

En effet, soient $v$ et $s$ tels que $\ell(v)<\ell(v s)$. Alors $\left(\bar{\pi}_{v}+\bar{\pi}_{v s}\right) \pi_{s}=\bar{\pi}_{v}+\bar{\pi}_{v s}$, $\bar{\pi}_{v} \pi_{s}=\bar{\pi}_{v}+\bar{\pi}_{v s} ;\left(\pi_{v s}-\pi_{v}\right) \bar{\pi}_{s}=-\pi_{v s}+\pi_{v}, \pi_{v} \bar{\pi}_{s}=\pi_{v s}-\pi_{v}$

Ce corollaire est utilisé de manière essentielle par Fokko du Cloux [Fo] pour implémenter l'ordre fort sur les groupes de Coxeter.

L'ordre fort peut s'obtenir à partir de la restriction de l'ordre aux sousensembles $W^{s}, s \in \mathcal{S}$, ainsi que l'établit le lemme suivant dû à Deodhar [De], qui généralise la définition géométrique de l'ordre fort donnée par Ehresmann [Eh] pour le groupe symétrique.

Lemme 3.3. - Soient $v, w \in W$. Alors $w \geq v$ ssi pour tout $s \in \mathcal{S}$, $p_{s}(w) \geq p_{s}(v)$.

Comme les morphismes $p_{s}$ sont des morphismes d'ordre, on peut écrire la propriété précédente

$$
w \geq v \Leftrightarrow \forall s \in \mathcal{S}, w \geq p_{s}(v)
$$

Plus symétriquement en la droite et la gauche, soient deux générateurs $r, s \in \mathcal{S}$ et ${ }^{r} W^{s}$ l'ensemble des bigrassmanniennes ayant recul $r$, descente $s$, ensemble auquel on adjoint l'identité. Cet ensemble est formé des éléments minimaux des classes $W(\mathcal{S} \backslash r) \backslash W / W(\mathcal{S} \backslash s)$.

On dispose d'une projection $p_{r, s}: W \rightarrow{ }^{r} W^{s}$ telle que

$$
p_{r, s}(w)=g \Leftrightarrow \partial_{w}=\partial_{w^{\prime}} \partial_{g} \partial_{w^{\prime \prime}}
$$

où $\partial_{g}$ est un facteur de $\partial_{w}$ de plus grande longueur parmi les $g \in{ }^{r} W^{s}$.

L'unicité d'un tel facteur provient de ce que les deux projections $W \rightarrow W /(\mathcal{S} \backslash s)$ et $W \rightarrow W(\mathcal{S} \backslash r) \backslash W$ commutent.

La propriété (3.4) implique

$$
w \geq v \Leftrightarrow \forall r, \forall s \in \mathcal{S}, w \geq p_{r, s}(v) .
$$

A tout élément $w \in W$, on associe l'élément

$$
p_{\mathcal{G}}(w):=\{g \in \mathcal{G}, g \leq w\} \subseteq 2^{\mathcal{G}},
$$

où $\mathcal{G}$ est l'ensemble des bigrassmanniennes. 
ThÉoréme 3.6. - Soit $(W, \mathcal{S})$ un groupe de Coxeter fini. Alors la base $B$ de $W$, en tant qu'ensemble muni de l'ordre fort, est contenue dans l'ensemble $\mathcal{G}$ des bigrassmanniennes.

Une bigrassmannienne $b$ ayant recul $r$ et descente $s$ appartient à la base ssi elle n'est pas le supremum d'un sous-ensemble de bigrassamnniennes $(\neq b)$ de mêmes descente et recul.

Preuve. - La propriété (3.5) implique que $p_{\mathcal{G}}$ soit un morphisme d'ordre. En outre $p_{\mathcal{G}}$ est injectif, car chaque élément $w$ est déterminé par l'ensemble des $p_{r, s}(w), r, s \in \mathcal{S}$, qui est un sous-ensemble de $p_{\mathcal{G}}(w)$. La caractérisation par minimalité de la base donnée en (2.4) implique que $\mathcal{G}$ contienne la base.

Les éléments de l'ensemble $W \backslash B$ sont les éléments de $W$ dont l'ensemble des rectrices est de cardinal $\neq 1$. Soit $g$ une bigrassmanienne de descente $s$ et recul $r$, et soit $\left\{b_{1}, \ldots, b_{k}\right\}$ l'ensemble de ses rectrices. Supposons que $k>1$. Alors tous les $b_{i}, i=1 \ldots k$, ont mêmes descente et recul que $g$. En effet, s'il existe $i$ tel que $b_{i}$ ait descente $s^{\prime} \neq s$, alors $b_{i}>b_{i} s^{\prime}$ et $w<w s^{\prime}$. Soient $b_{k+1}, \ldots, b_{h}$ les rectrices de $b_{i} s^{\prime}$. La condition $w \geq b_{i}$ est équivalente à $w \geq b_{i} s^{\prime}$, elle même équivalente à $w \geq b_{k+1}, \ldots, w \geq b_{h}$. L'élément $w$ est donc le supremum de l'ensemble $\left\{b_{1}, \ldots, b_{i-1}, b_{i+1}, \ldots, b_{h}\right\}$, ce qui est impossible puisque cet ensemble ne contient pas la rectrice $b_{i}$.

Réciproquement, si une bigrassmannienne dans ${ }^{r} W^{s}$ est le supremum de $\left\{g_{1}, \ldots, g_{k}\right\} \subseteq{ }^{r} W^{s}, k>1$, alors elle est le supremum de leurs rectrices qui appartiennent toutes à ${ }^{r} W^{s}$. Ainsi donc une bigrassmannienne de descente $s$ et recul $r$ n'appartient pas à la base ssi elle est le supremum d'un ensemble de cardinal $>1$ de bigrassmanniennes dans ${ }^{r} W^{s}$ deux à deux incomparables

Nous détaillons par la suite les bases des groupes classiques de type $A_{n}$ et $B_{n}$.

4. Ehresmannoedre. - L'ordre fort, dit aussi de Bruhat, sur le groupe symétrique est usuellement défini comme la clôture transitive des relations de consécutivité

$$
\{\nu \rightarrow \mu\} \Leftrightarrow\{\exists \tau: \mu=\nu \tau, \ell(\mu)=\ell(\nu)+1\}
$$

où $\nu, \mu$ sont deux permutations et $\tau$ une transposition, plutôt que par sous-mots des décompositions réduites comme au paragraphe 2 .

Le groupe symétrique, muni de cet ordre, est dit Ehresmannoedre. Nous allons voir que sa base consiste en l'ensemble des bigrassmanniennes. La base est donc exactement l'intersection des bases pour les ordres faibles droit et gauche. 
La définition originelle d'Ehresmann [Eh] de l'ordre sur le groupe symétrique repose quant à elle sur la comparaison des ensembles.

Deux sous-ensembles $A$ et $B$ d'un ensemble totalement ordonné sont comparables : $A \leq B$ ssi il existe un morphisme injectif de $A$ dans $B$. Une suite d'ensembles se représente planairement par une suite de colonnes décroissantes justifiée par le haut, ces colonnes étant les ensembles réordonnés composant la suite. La suite d'ensembles est une chaîne (i.e. est croissante) si l'objet planaire est un contretableau, i.e. si les colonnes sont de longueurs croissantes, et si toutes les lignes sont croissantes de gauche à droite.

Etant donnés deux contretableaux $t$ et $t^{\prime}$ de même forme, on dit que $t \leq t^{\prime}$ ssi chaque composante de $t$ est inférieure à la composante de $t^{\prime}$ situéee au même endroit. On notera que la restriction de l'ordre au sousensemble des colonnes coïncide bien avec l'ordre sur les ensembles sousjacents.

Les facteurs gauches successifs d'une permutation $\mu$ sont une suite d' ensembles emboîtés (un drapeau ). Le contretableau associé est dit clef $f(\mu)$.

Ainsi $\mu=2413$ a pour clef

$\begin{array}{llll}2 & 4 & 4 & 4 \\ & 2 & 2 & 3 \\ & & 1 & 2 \\ & & & 1\end{array}$

Le lemme suivant montre que l'ordre fort coïncide avec la comparaison des clefs, qui est la définition choisie par Ehresmann.

LEMME 4.1. - $\mu \leq \nu$ ssi clef $(\mu) \leq \operatorname{clef}(\nu)$

Chaque colonne de la clef d'une permutation détermine une permutation grassmannienne. Ansi la clef de $\mu=2413$ correspond-elle à la suite des grassmanniennes 2143, 2413, 1243, 1234. La comparaison de deux clefs correspond donc à la comparaison de grassmanniennes ayant même descentes. C'est aussi la comparaison des partitions obtenues en soustrayant $1,2 \ldots$ aux colonnes de la clef.

De manière symétrique, la comparaison des inverses des permutations peut se décrire en coupant l'alphabet $\{1, \ldots, n\}$ en deux intervalles. Soit en effet $\{x<y\}$ un alphabet à deux lettres, et $p_{h}$ la projection de $\mathfrak{S}(n)$ (considéré comme un ensemble de mots en $1, \ldots, n$ ) sur le monoïde $\{x, y\}^{*}$ induite par le morphisme

$$
i \rightarrow x \text { si } i \leq h, i \rightarrow y \text { si } i>h .
$$

L'ordre sur les mots en $\{x, y\}$ se définit en comparant les degrés en $y$ des facteurs gauches respectifs de même longueur des deux mots :

$$
v \leq w \text { si }\left\{v=v^{\prime} v^{\prime \prime}, w=w^{\prime} w^{\prime \prime},\left|v^{\prime}\right|=\left|w^{\prime}\right|\right\} \Rightarrow\left|v^{\prime}\right|_{y}=\left|w^{\prime}\right|_{y} .
$$


On a alors

LEMME 4.2. - $\mu \leq \nu$ ssi pour tout $h$, tout $k$,

$$
\left|p_{h}\left(\mu_{1} \cdots \mu_{k}\right)\right|_{y} \leq \mid p_{h}\left(\left.\nu_{1} \cdots \nu_{k}\right|_{y}\right.
$$

Ainsi $2143 \leq 4132$, car, écrivant $\{1 \ldots i \mid i+1 \ldots n\}$ pour la projection $1, \ldots, i \rightarrow x, i+1, \ldots, n \rightarrow y$, on a :

pour $\{1 \mid 234\}$, yxyy $\leq$ yxyy; pour $\{12 \mid 34\}$, xxyy $\leq$ yxyx; pour $\{123 \mid 4\}$, $x x y x \leq y x x x$.

Le lemme 4.2 n'est autre que l'explicitation des projections $p_{h k}$ (vues en 3.5) de $\mathfrak{S}(n)$ sur l'ensemble des éléments de longueur minimum des classes $\mathfrak{S}(h) \times \mathfrak{S}(n-h) \backslash \mathfrak{S}(n) / \mathfrak{S}(k) \times \mathfrak{S}(n-k)$.

On peut maintenant rétablir la symétrie (pour l'inversion des permutations) de l'Ehresmannoedre, qu'avait mise à mal la comparaison des clefs de $\mu$ et $\nu$.

Voyons tout d'abord la comparaison d'une permutation et d'une bigrassmannienne. Comme une bigrassmannienne $b$ de $\mathfrak{S}(n)$ est une permutation qui a pour code un rectangle $0^{r_{0}} r_{2}^{r_{1}} 0^{r_{3}}$, avec $r_{0}+r_{1}+r_{2}+r_{3}=n$, $r_{1} \geq 1, r_{2} \geq 1$, on la désignera plutôt par le symbole $b=\left[r_{0}, r_{1}, r_{2}, r_{3}\right]$. Alors

Lemme 4.3. - Une permutation $\mu$ est supérieure à une bigrassmannienne $b=\left[r_{0}, r_{1}, r_{2}, r_{3}\right]$ ssi il y a au moins $r_{1}$ valeurs $>r_{0}+r_{2}$ dans le facteur gauche de longueur $r_{0}+r_{1}$ de $\mu$.

Preuve. - On utilise le critère 4.2. Pour la coupure de l'alphabet $\left\{1 \cdots r_{0}+\right.$ $\left.r_{2} \mid r_{0}+r_{2}+1 \cdots n\right\}$, le mot $x^{r_{0}} y^{r_{1}} x^{r_{2}} y^{r_{3}}=p_{r_{0}+r_{2}}(b)$ est clairement le minimum des $p_{r_{0}+r_{2}}(\mu)$, pour $\mu \geq b$.

On peut tout autant optimiser la négation $N E G$ de la condition $\mu \geq b$ ( nous prions le lecteur de se reporter à la dernière partie de l'annexe où est traitée la notion de clivage). En effet, le mot $y^{r_{1}-1} x^{r_{0}+1} y^{r_{3}+1} x^{r_{2}-1}$ est le maximum des $p_{r_{0}+r_{2}}(\zeta), \zeta \nsupseteq b$, et a pour maximum dans sa fibre la permutation

$c_{\left[r_{0} r_{1} r_{2} r_{3}\right]}=$

$n \cdots\left(n-r_{1}+2\right)\left(r_{0}+r_{2}\right) \cdots r_{2}\left(r_{0}+r_{2}+r_{3}+1\right) \cdots\left(r_{0}+r_{2}+1\right)\left(r_{2}-1\right) \cdots 1$.

Ainsi donc les bigrasmanniennes constituent la base de l'Ehresmannoedre, car à chaque bigrassmannienne $b$ correspond bijectivement une cobigrassmannienne qui est le Sup du complémentaire de l'intervalle supérieur $[b \leq]$. En d'autres termes, on a le théorème suivant. 
ThÉORÈme 4.4. - Le treillis enveloppant du groupe symétrique est distributif et a pour générateur les bigrassmanniennes.

Plus précisément, soit $\left[r_{0}, r_{1}, r_{2}, r_{3}\right] \in \mathbb{N}^{4}, r_{1}, r_{2} \geq 1, r_{0}+r_{1}+r_{2}+r_{3}=$ $n$. Alors le groupe symétrique $\mathfrak{S}(n)$ clive en les deux intervalles $[b \leq]$ et $[\leq c]$, où $b$ et $c$ sont les bigrassmannienne et cobigrassmannienne codées $\operatorname{par}\left[r_{0}, r_{1}, r_{2}, r_{3}\right]$.

Une permutation $\mu$ appartient à l'un ou l'autre de ces deux intervalles selon que la composante de sa clef de coordonnées $r_{0}+r_{1}, r_{1}$ ( pour les axes $\downarrow \rightarrow$ ) est strictement supérieure à $r_{0}+r_{2}$ ou non.

Pour $\mathfrak{S}(4)$, l'ensemble $B$ des bigrassmanniennes est :

$$
\{2134,1324,1243,1342,1423,2314,3124,2341,4123,3412\}
$$

La restriction de la relation d'incidence à $\mathfrak{S}(4) \times B$, dont les lignes sont les projections par $\beta$ des éléments sur la base $B$ est représentée par la matrice:

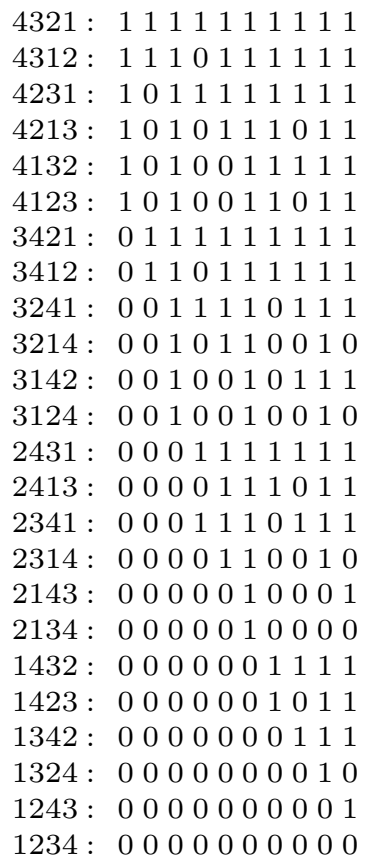

Enfin, les dix clivages correspondant aux bigrassmanniennes sont :

$$
\begin{aligned}
\mathfrak{S}(4)=[2134 \leq] \cup[\leq 1432] & =[1324 \leq] \cup[\leq 2143]=[1243 \leq] \cup[\leq 3214] \\
=[1342 \leq] \cup[\leq 4213] & =[1423 \leq] \cup[\leq 3241]=[2314 \leq] \cup[\leq 4132] \\
& =[3124 \leq] \cup[\leq 2431] \\
& =[2341 \leq] \cup[\leq 4312]=[4123 \leq] \cup[\leq 3421] \\
& =[3412 \leq] \cup[\leq 4231]
\end{aligned}
$$


Par ailleurs, les bigrassmanniennes étant codées bijectivement par les vecteurs $\left[r_{0}, r_{1}, r_{2}, r_{3}\right] \in \mathbb{N}^{4}, r_{1}, r_{2} \geq 1$, la fonction génératrice du nombre de bigrassmanniennes, pour $n$ variable, est

$$
\sum_{n \geq 2} z^{n-2} \operatorname{card}(\operatorname{Base}(\mathfrak{S}(n)))=(1-z)^{-4}
$$

L'image d'une permutation par la projection $p_{i j}$ vue en 3.5 est soit l'identité, soit une bigrassmannienne $b$ ayant recul $i$ et descente $j$ (i.e. $b$ a le sous-mot $i+1 \ldots i$ et $\left.b_{j}>b_{j+1}\right)$. En outre, si $b$ est une bigrassmannienne de recul $i$ et descente $j$, alors $b \leq \mu \Rightarrow b \leq p_{i j}(\mu)$. Les rectrices d'une permutation $\mu$ sont donc les éléments maximaux de l'ensemble $\left\{p_{i j}(\mu)\right\}_{1 \leq i, j \leq n-1}$. Il existe un algorithme pour déterminer ces rectrices, sans calculer l'ensemble précédent, qui repose sur le lemme suivant:

Lemme 4.5. - Il y a bijection entre les rectrices d'une permutation $\mu$ et les triples d'entiers $i \leq j<j+1 \leq k$ tels que

$$
\mu_{j+1} \leq \mu_{k}<\mu_{i}=1+\mu_{k} \leq \mu_{j} .
$$

$A$ un tel triple correspond la rectrice $b=\left[r_{0}, r_{1}, r_{2}, r_{3}\right]$, où $r_{0}=\operatorname{card}\left\{h<j: \mu_{h}<\mu_{k}\right\}, r_{1}=j-r_{0}, r_{2}=\mu_{k}-r_{0}$.

Nous laissons au lecteur le soin de vérifier que la bigrassmannienne associée au couple : descente $\left(\mu_{j}>\mu_{j+1}\right)$, recul $\left(\mu_{k}+1 \ldots \mu_{k}\right)$ est bien maximale sous les hypothèses du lemme 4.5.

Graphiquement, une rectrice $b$ correspond à une configuration

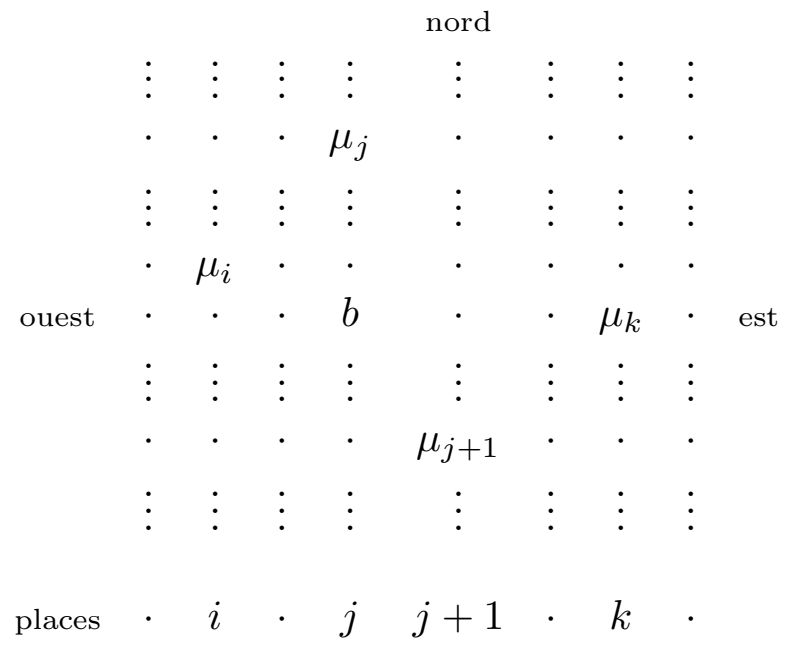

pour les axes de coordonnées cartésiens $\uparrow \rightarrow$, le couple $\left(\mu_{k}, j\right)$ étant dit les coordonnées de la rectrice. 
Outre l'ordre de Bruhat, on peut lire certaines propriétés des permutations sur l'ensemble de leurs rectrices. Par exemple, la proposition suivante décrit l'appartenance à un sous-groupe de Young, sous des conditions que l'on peut qualifier de géographiques car elles ne font intervenir que les coordonnées $r_{0}+r_{1}, r_{0}+r_{2}$ des rectrices.

Proposition 4.6. - Soit $\mu$ une permutation, $R=R^{\prime} \uplus R^{\prime \prime}$ une décomposition de l'ensemble de ses rectrices $\left(R^{\prime}, R^{\prime \prime} \neq \emptyset\right)$.

Soient $n_{1}, n_{2}$ deux entiers tels que

$$
\begin{aligned}
r_{0}+r_{1} & \leq n_{1} \quad \& \quad r_{0}+r_{2} \leq n_{2} \text { si }\left[r_{0}, r_{1}, r_{2}, r_{3}\right] \in R^{\prime} \\
& >n_{1}
\end{aligned}
$$

Alors il existe un entier $n_{3}$ tel que l'on ait les mêmes relations en remplaçant $\left(n_{1}, n_{2}\right)$ par $\left(n_{3}, n_{3}\right)$, et $\mu$ appartient au sous-groupe $\mathfrak{S}\left(n_{3}\right) \times$ $\mathfrak{S}\left(n-n_{3}\right)$.

Preuve. - Soient $n_{4}$ et $n_{5}$ les deux entiers : $n_{4}=\operatorname{Max}\left(\left\{\mu_{j}:\right.\right.$ il existe une rectrice de $\mu$ dans $R^{\prime}$ avec $\left.\left.r_{0}+r_{1}=j\right\}\right) ; n_{5}=\operatorname{Max}(\{k j$ : il existe une rectrice de $\mu$ dans $R^{\prime}$ avec $\left.r_{0}+r_{2}=\mu_{k}\right\}$ ) .

Par maximalité de $n_{4}=\mu_{j}$, on a $\mu_{j}>\mu_{j+1}$. D'après le lemme 4.5 et l'hypothèse 4.6 , on a $\mu_{j+1}<n_{2}$. Pour les mêmes raisons, on a aussi $\mu_{k}>n_{4}$ pour tout $h>n_{1}$, ce qui entraine que les zones $\star$ ne contiennent pas de point $\left(i, \mu_{i}\right)$ :

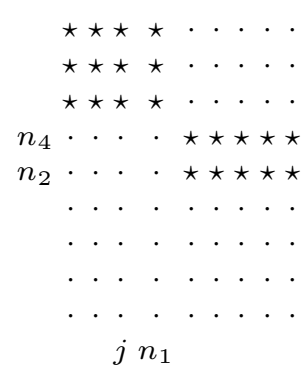

Le même argument s'applique à $n_{5}$ grâce à la symétrie $\mu \rightarrow \mu^{-1}$. Le domaine permis pour les points $\left(i, \mu_{i}\right)$ est donc finalement (zone ".") : 


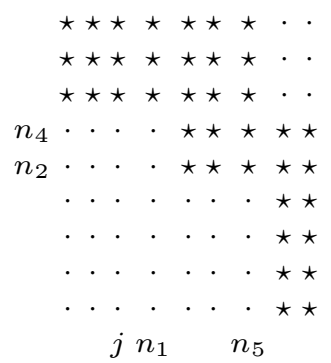

Ceci montre que $n_{3}=\operatorname{Max}\left(n_{4}, n_{5}\right)$ satisfait l'énoncé 4.6, et que $i \leq n_{3} \Rightarrow \mu_{i} \leq n_{3}$

5. Triangles. - On peut identifier chaque permutation $\mu$ à sa clef $K(\mu)$. La comparaison d'une permutation $\mu$ et d'une bigrassmannienne $b=\left[r 0, r_{1}, r_{2}, r_{3}\right]$ se réduit à tester une seule composante de $K(\mu)$, d'après le théorème 4.4 :

$$
\mu \geq b \text { ssi } K(\mu)_{r 0+r 1, r_{1}}>r_{0}+r_{2} .
$$

Le Sup ou l' Inf, dans le treillis enveloppant, d'une famille $\{\mu\}$ de permutations se calcule aisément, étant le Sup ou l'Inf composante à composante des $\{K(\mu)\}$.

Plus précisément, un contretableau $t$ de forme $12 \cdots n$ est le sup de la famille $\{\mu\}$ ssi

$$
\forall i, j: 1 \leq i \leq j \leq n, t_{i j}=\operatorname{Sup}_{\mu}\left\{K(\mu)_{i j}\right\} .
$$

Il est aisé de contrôler qu'un tel contre-tableau vérifie, pour chaque soustriangle élémentaire (i.e. triple de composantes adjacentes de coordonnées respectives $(i, j),(i, j+1)$ et $(i+1, j+1)$ pour les axes $\downarrow \rightarrow)$ les inégalités suivantes

$$
\underset{x}{y z} \Rightarrow\{x<z \& x \leq y \& y \leq z\} \text {. }
$$

Réciproquement, tout contre-tableau de forme $12 \cdots n$ vérifiant les conditions 5.2 ( que l'on appelera triangle) est le Sup de la famille de bigrassmanniennes déterminée par chacune de ses composantes :

$$
t_{i j} \rightarrow b=\left[j-i, i, t_{i j}+i-j-1, n-t_{i j}-i+1\right]
$$

On a donc 
LEMME 5.4. - Le treillis enveloppant du groupe symétrique $\mathfrak{S}(n)$ est isomorphe au treillis des triangles de forme $12 \cdots n$, remplis avec des lettres dans $\{1,2, \ldots, n\}$.

D'après les travaux de Mills, Robbins \& Rumsey [MRR] les triangles (qu'ils appellent triangles monotones) sont en bijection avec les matrices alternantes, dont le cardinal, pour l'ordre $n$, est

$$
\prod_{0}^{n-1}(3 k+1) ! /(n+k) !
$$

Cette formule, qu'ils conjecturaient, a depuis été prouvée.

Pour $\mathfrak{S}(3)$, le treillis enveloppant comprend un seul élément qui n'est pas une permutation : c'est le Sup de $213 \leftrightarrow{ }_{1}^{22}$ et $132 \leftrightarrow{ }_{1}^{13}$, ainsi que l'Inf de $231 \leftrightarrow{ }_{2}^{23}$ et $312 \leftrightarrow{ }_{1}^{33}$. C'est donc ${ }_{1}^{23}$ qui correspond à la seule matrice alternante d'ordre trois qui ne soit pas une permutation : $\begin{array}{cccc}0 & 1 & 0 \\ 1 & -1 & 1\end{array}$.

Le treillis enveloppant de $\mathfrak{S}(4)$ est constitué de 42 triangles, dont nous énumérons les 18 qui ne sont pas des clefs:

$\begin{array}{rrrrrrrrrr}233 & 134 & 234 & 234 & 334 & 234 & 334 & 234 & 334 \\ 12 & 12 & 12 & 22 & 12 & 13 & 22 & 23 & 23 \\ 1 & 1 & & 1 & 1 & 1 & 1 & 1 & 1 & 1 \\ & & & & & & & & \\ & & & & & & & & \\ & & & & & & & & & \\ 244 & 344 & 244 & 344 & 244 & 344 & 344 & 344 & 444 \\ 12 & 12 & 13 & 22 & 23 & 13 & 23 & 23 & 23 \\ 1 & 1 & 1 & 1 & 1 & 1 & 1 & 2 & 1\end{array}$

L'application 5.3 décrit, pour un contre-tableau $t$, une famille de bigrassmanniennes dont $t$ est le Sup. Les éléments maximaux de cet ensemble de bigrassmanniennes, c'est-à-dire les rectrices du triangle, correspondent bijectivement aux composantes $t_{i j}$ telles que l'image de $t$ par $t_{i j} \rightarrow t_{i j}-1$ soit encore un triangle.

Ces composantes seront appelées points essentiels du triangle; un triangle est donc le Sup des bigrassmanniennes correspondant à ses points essentiels et tout ensemble de composantes dont le triangle est le Sup contient les points essentiels. Dans le cas où $t$ est la clef d'une permutation $\mu$, les points essentiels ont été définis par Fulton $[\mathrm{Fu}]$ ( en terme de la fonction de rang de la matrice représentant la permutation). 
Un triangle sera dit vexillaire ssi l'ensemble de ses points essentiels $\mathcal{E}$ vérifie la condition suivante (qui est géographique au sens donné plus haut à ce terme):

$$
\forall(i, j) \in \mathcal{E} \Rightarrow\left\{\left(i^{\prime}, j^{\prime}\right): i^{\prime} \leq i, j^{\prime}>j\right\} \cap \mathcal{E}=\emptyset
$$

En d'autres termes, il n'y a pas de point essentiel dans le cadran nord-est d'un point essentiel (le domaine d'exclusion comprend le demi axe-est).

Dans le cas d'une permutation vexillaire $\mu$, cette définition, appliquée à la clef de $\mu$, correspond à l'une des nombreuses caractérisations des permutations vexillaires (cf. $[\mathrm{Fu}])$.

6. Pavés. - On a remarqué que le clivage associé à une transposition simple $\sigma$ induit le clivage de tout intervalle $[\leq \mu]$. Cet idéal se décompose en un idéal $[\leq \nu]$ (où $\nu$ est la permutation maximum $\nu \leq \mu$ dont aucune décomposition réduite ne contient $\sigma$ ) et son complémentaire $[\leq \mu] \cap[\sigma \leq]$.

Nous donnons dans ce paragraphe une décomposition du groupe symétrique transversale au clivage précédent.

Pour ce faire, considérons l'algèbre des différences divisées isobares.

A tout ensemble $\mathcal{J} \subset\{1, \ldots, n-1\}$ correspond le groupe parabolique $\mathcal{P}_{\mathcal{J}}$ engendré par les transpositions simples $\left\{\sigma_{i} \mid i \in\{1 \ldots n-1\} \backslash \mathcal{J}\right\}$, ainsi que la projection $p_{\mathcal{J}}$ du monoide engendré par les $\pi_{i}, i \in\{1 \ldots n-1\}$ sur le monoide engendré par les $\pi_{i}, i \notin \mathcal{J}$ déterminée par $\pi_{j} \rightarrow 1$ pour tous les $j \in \mathcal{J}$.

Cette projection est clairement compatible aux relations de Coxeter, ainsi qu'aux relations $\pi_{i}^{2}=\pi_{i}$ et donc l'image des $\pi_{\mu}$ est bien définie.

Définition. - Soit $k$ un entier, $1 \leq k \leq n-1$. Un $k$-pavé est une classe d'équivalence pour la relation

$$
\mu \sim_{k} \nu \Leftrightarrow p_{k}\left(\pi_{\mu}\right)=p_{k}\left(\pi_{\nu}\right)
$$

Deux permutations $\mu, \nu$ appartiennent au même $k$-pavé ssi, pour deux décompositions réduites quelconques $\mu=\sigma_{i} \sigma_{j} \cdots \sigma_{h}$ et $\nu=\sigma_{l} \cdots \sigma_{m}$, on a

$$
p_{k}\left(\pi_{i} \pi_{j} \cdots \mu_{h}\right)=p_{k}\left(\pi_{l} \cdots \pi_{m}\right) .
$$

Par exemple, le groupe $\mathfrak{S}(4)$ se décompose en six 1-pavés : [1432, 4321], [1342, 3241], [1423, 4213], [1324, 3214], [1243, 2143], [1234, 2134], qui sont des intervalles booléens de cardinaux respectifs 8,4,4,4,2,2 .

Des pavés plus généraux apparaissent pour $k \neq 1, n-1$. 
Définition. - Soit $\mu$ une permutation et $k \leq n-1$. Les places $k$ saillantes gauches de $\mu$ sont les indices $1 \leq i \leq k$ pour lesquels $\mu_{i}=\max \left(\left\{\mu_{i}, \ldots, \mu_{k}\right\}\right)$; les places $k$-saillantes droites de $\mu$ sont les indices $k<j \leq n$ pour lesquels $\mu_{j}=\min \left(\left\{\mu_{k+1}, \ldots, \mu_{j}\right\}\right)$.

Proposition 6.1. - Soit $k$ un entier $\leq n-1, \nu$ une permutation dans $\mathcal{P}_{k}=\mathfrak{S}(k) \times \mathfrak{S}(n-k)$. Soit $\zeta$ la permutation obtenue à partir de $\nu$ en permutant circulairement les points saillants de $\nu$ de sorte à en faire une suite décroissante.

Alors le k-pavé contenant $\nu$ est égal à l'intervalle $[\nu, \zeta]$ et est isomorphe à l'idéal de la bigrassmannienne $[0, r, p, 0]$ de $\mathfrak{S}(p+r)$, où $p$ est le nombre de points saillants gauches de $\nu, r$ le nombre de points saillants droits.

Une permutation $\mu$ appartient au pavé de $\nu$ ssi $\zeta_{i}=\nu_{i} \Rightarrow \mu_{i}=\nu_{i}$ et l'image de $\mu$ par la projection de $\mathfrak{S}(n)$ sur $\mathfrak{S}(p+r)$ qui consiste en l'effacement des valeurs communes à $\nu$ et $\zeta$, est supérieure (pour l'ordre de Bruhat) à l'image de $\nu$.

Ainsi, pour $k=5$ et

$$
\begin{array}{cccccccccccccc}
\nu & = & \mathbf{5} & 3 & \mathbf{4} & 1 & \mathbf{2} & \mathbf{1 1} & \mathbf{9} & 12 & \mathbf{7} & 8 & \mathbf{6} & 10 \\
& 1 & 2 & 3 & 4 & 5 & 6 & 7 & 8 & 9 & 10 & 11 & 12
\end{array}
$$

$\begin{array}{lllllllllllll}\text { places } & 1 & 2 & 3 & 4 & 5 & 6 & 7 & 8 & 9 & 10 & 11 & 12\end{array}$

( les point saillants sont en gras), le maximum du pavé est

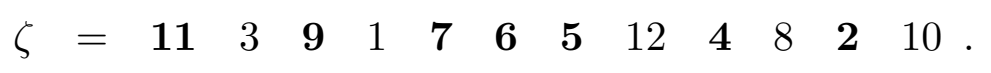

Une permutation $\mu$ appartient au pavé ssi $\mu_{2}=3, \mu_{4}=1, \mu_{8}=12$, $\mu_{12}=10$ et $\left(\mu_{1}, \mu_{3}, \mu_{5}, \mu_{6}, \mu_{7}, \mu_{9}, \mu_{11}\right) \geq(5,4,2,11,9,7,6)$.

Preuve. - La permutation $\nu$ est le produit direct des deux permutations $\nu^{\prime}=\nu_{1} \ldots \nu_{k} k+1 \ldots n$ et $\nu^{\prime \prime}=1 \ldots k \nu_{k+1} \ldots \nu_{n}$. Une permutation $\mu$ est dans la fibre de $\nu$ ssi $p_{k \ldots n-1}(\mu)=\nu^{\prime}$ et $p_{1 \ldots k}(\mu)=\nu^{\prime \prime}$. Or le code de $p_{k \ldots n-1}(\mu)$ est l'inf du code de $\mu$ et du vecteur $[k-1, \ldots, 1,0, \ldots, 0]$, ainsi qu'on le voit en considérant le décomposition réduite de $\mu$ associée au code (i.e. le code considéré est le vecteur $\left[\inf \left(c_{1}, k-1\right), \ldots, \inf \left(c_{k}, 0\right), \ldots, 0\right]$, où $\left[c_{1}, \ldots, c_{n}\right]$ est le code de $\left.\mu\right)$.

Il s'ensuit que la condition nécessaire et suffisante que $\mu$ soit dans la fibre de $\nu$ est que

- l'inf du code de $\mu$ et $[k-1 \ldots 0 \ldots 0]$ est égal au code de $\nu^{\prime}$

- l'inf du code de $\omega \mu \omega$ et $[n-k-1 \ldots 0 \ldots 0]$ est égal au code de $\omega \nu^{\prime \prime} \omega$.

Il n'est pas difficile de vérifier que $\zeta$ satisfait à ces deux conditions.

Comme les morphismes de spécialisation $p_{\mathcal{J}}$ sont des morphismes d'ordre (i.e. $\mu \leq \eta \Rightarrow p_{\mathcal{J}}(\mu) \leq p_{\mathcal{J}}(\eta)$ ), on en déduit que l'intervalle $[\nu, \zeta]$ est contenu tout entier dans une seule fibre. 
Nous épargnerons au lecteur la vérification que toute permutation appartient à un et un seul de ces intervalles.

Par ailleurs, l'intervalle $[\nu, \zeta]$ a pour image dans $\mathfrak{S}(p+r)$, après effacement des points fixes et retour à l'alphabet $\{1,2, \ldots, p+r\}$ l'intervalle $[(p \ldots 1),(p+r \ldots p+1)]$. L'image de cet intervalle, par multiplication à gauche par $\omega$, est l'intervalle $[(1 \ldots p),(p+1 \ldots p+r)]$ qui n'est autre que l'intervalle inférieur de la bigrassmannienne $[0, r, p, 0]$

L'énumération des pavés isomorphes à l'idéal de la bigrassmannienne $[0, r, p, 0]$ ( disons de type $[0, r, p, 0]$ ) est donné par le lemme suivant, en désignant par $\Lambda^{i}(k)$ le nombre de Stirling égal à la $i$-ème fonction élémentaire des entiers $0,1, \ldots, k-1$.

Lemme 6.2. - Le nombre de $k$-pavés de type $[0, r, p, 0]$ est égal au produit des nombres de Stirling $\Lambda^{k-p}(k) \Lambda^{n-k-r}(n-k)$

Preuve. - Soit $n(k, r, p)$ le nombre de pavés de type $[0, r, p, 0]$. La projection "effacement de la lettre 1" :

$$
\mathfrak{S}(n) \ni \mu \rightarrow \mu \backslash 1 \in \mathfrak{S}(n-1)
$$

donne une récurrence sur le nombre de pavés.

En effet, soit $\nu$ le minimum d'un $k$-pavé. Si $\nu_{k}=1$, alors le pavé est de type $[0, r, p, 0]$ ssi le pavé contenant $\nu \backslash 1$ est de type $[0, r, p-1,0]$.

Si $\nu_{k} \neq 1$, l'effacement de 1 donne un pavé isomorphe. Comme il y a $k-1$ permutations $\mu$ qui se projettent sur $\nu \backslash 1$, et telles que $\nu_{k} \neq 1$, on a la récurrence $n(k, r, p)=(k-1) n(k-1, r, p)+n(k-1, r, p-1) \square$

Exemple. - Les fibres de la projection $p_{3}: \mathfrak{S}(7) \rightarrow \mathfrak{S}(3) \times \mathfrak{S}(4)$ sont les bigrassmanniennes suivantes. On regroupe les pavés de type $[0, r, p, 0]$ et $[0, p, r, 0]$ qui sont isomorphes, et on écrit $\Lambda^{i j}$ pour $\Lambda^{i}(3) \Lambda^{j}(4)$ :

$\begin{array}{cccccccccc}{[p, r]} & {[11]} & {[12]} & {[13]} & {[14]} & {[22]} & {[23]} & {[24]} & {[33]} & {[34]} \\ \text { card } & 12 & 40 & 18 & 2 & 33 & 29 & 3 & 6 & 1 \\ = & \Lambda^{23} & \Lambda^{22}+\Lambda^{13} & \Lambda^{21}+\Lambda^{03} & \Lambda^{20} & \Lambda^{12} & \Lambda^{02}+\Lambda^{11} & \Lambda^{10} & \Lambda^{01} & \Lambda^{00}\end{array}$

Molev [Mo] a utilisé une décomposition du groupe symétrique en intervalles booléens pour développer le déterminant de Sklyanin et décrire le centre de l'algèbre enveloppante dans le cas des groupes orthogonaux et symplectiques. Quoique le nombre des pavés soit encore un nombre de Stirling, cette décomposition est différente de celle que nous venons d'exposer.

Ayant décomposé le groupe symétrique en pavés, il nous reste à décrire ceux-ci en tant qu'intervalles pour l'ordre de Bruhat. Nous nous contenterons de donner la fonction génératrice du nombre d'éléments dans l'intervalle, classés par nombre d'inversions, autrement dit le Polynôme de Poincaré de l'intervalle. 
Soit une bigrassmannienne $b=[0, r, p, 0]$. Notons $G(r, p)$ le polynôme de Poincaré $:=\sum_{\mu<b} z^{\ell(\mu)}$ de $b$.

Nous voulons décrire l'intervalle inférieur de $b$. Celle dernière a une décomposition réduite canonique que l'on peut écrire planairement dans un rectangle (lisant par colonnes, de gauche à droite):

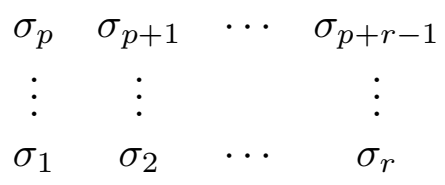

Comme l'intervalle se calcule à l'aide de (3.2), c'est-à-dire de la décomposition des produits de $\pi_{i}$ dans la base des $\bar{\pi}_{\mu}$, le polynôme de Poincaré $G(r, p)$ est obtenu par récurrence à partir du lemme suivant.

Lemme 6.3. - Soient $p$ et $r$ deux entiers. Alors on a l'identité

$$
\begin{aligned}
& \begin{array}{lllllllll}
\pi_{p} & \pi_{p+1} & \cdots & \pi_{p+r-1} & \cdot & \bar{\pi}_{p-1} & \bar{\pi}_{p} & \cdots & \bar{\pi}_{p+r-1}
\end{array} \\
& \begin{array}{ccccc}
\pi_{p-1} & \pi_{p} & \cdots & \pi_{p+r-2} & \bar{\pi}_{p+r-1} \\
\vdots & \vdots & & \vdots & \vdots
\end{array}
\end{aligned}
$$

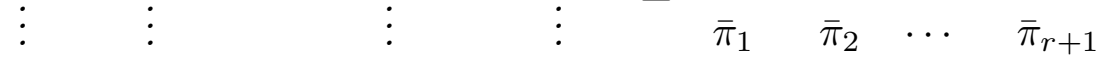

$$
\begin{aligned}
& \begin{array}{lllllllll}
\pi_{1} & \pi_{2} & \ldots & \pi_{r} & \bar{\pi}_{r+1} & \cdot & \pi_{1} & \cdots & \pi_{r}
\end{array}
\end{aligned}
$$

Preuve. - Ecrivons $i$ pour $\pi_{i}$, $i^{\prime}$ pour $\bar{\pi}_{i}$. On a, pour deux entiers consécutifs quelconques,

$$
434^{\prime}=43^{\prime} 4^{\prime}=3^{\prime} 4^{\prime} 3
$$

et donc, pour deux lignes,

$$
\begin{aligned}
& \begin{array}{lllllll}
2 & 3 & 4 & \cdot & 2 & 3 & 2
\end{array} \\
& 1234^{\prime}=1 \quad 2 \quad 3^{\prime} \quad 4^{\prime}=1 \quad 2^{\prime} \quad 3^{\prime} \quad 4^{\prime}=1^{\prime} \quad 2^{\prime} \quad 3^{\prime} \quad 4^{\prime} \\
& \begin{array}{llllll}
3 & 2 & 3 & 1 & 2 & 3
\end{array}
\end{aligned}
$$

Finalement, pour un nombre quelconque de lignes,

$$
\begin{aligned}
& \begin{array}{lllllllllllll}
3 & 4 & 5 & \cdot & 2^{\prime} & 3^{\prime} & 4^{\prime} & 5^{\prime} & . & 2^{\prime} & 3^{\prime} & 4^{\prime} & 5^{\prime}
\end{array} \\
& 2345^{\prime}=\cdot 2 \quad 3 \quad 4 \quad \cdot=1^{\prime} \quad 2^{\prime} \quad 3^{\prime} \quad 4^{\prime} \text {, } \\
& \begin{array}{llllllllllllll}
1 & 2 & 3 & 4^{\prime} & \cdot & 1 & 2 & 3 & 4^{\prime} & & \cdot & 1 & 2 & 3
\end{array}
\end{aligned}
$$

ce qui est bien le lemme

On intialise la récurrence sur les polynômes de Poincaré en écrivant que la dernière colonne de l'expression planaire de $\pi_{b}$, disons $\pi_{6} \pi_{5} \pi_{4} \pi_{3}$ est égale à 


$$
\pi_{6} \pi_{5} \pi_{4} \pi_{3}=\left(1+\overline{\pi_{6}}\right)\left(1+\overline{\pi_{5}}\right)\left(1+\overline{\pi_{4}}\right)\left(1+\overline{\pi_{3}}\right)
$$

Par application répétée de 6.3, en regroupant les termes et en employant les notations des $\lambda$-anneaux ( les fonctions $S^{i}(r+x)$ sont les coefficients de la série en $z 1 /(1-z x)(1-z)^{r}$, i.e.

$\left.1 /(1-z x)(1-z)^{r}=S^{0}(r+x)+z S^{1}(r+x)+z^{2} S^{2}(r+x)+\ldots\right)$, on obtient

Proposition 6.4. - Soient $r, p$ des entiers $\geq 1$ et $Z=z^{r+1}$. Alors

$G(r+1, p)=\left(S^{p-1}(1+z)+z^{p}\right) G(r, p)+Z S^{p-2}(2+z) G(r, p-1)+$ $Z^{2} S^{p-3}\left((3+z) G(r, p-2)+\cdots+Z^{p-2} S^{1}(p-1+z) G(r, 2)+Z^{p-1} S^{0}(r, 1)\right.$

Le cas initial $G(r, 1)$, c'est à dire de l'idéal d'un cycle $b=(2, \ldots, r+1,1)$ est donné par la spécialisation $\pi_{i}=1+z \forall i$. En effet, $\pi_{b}=(1+$ $\left.\overline{\pi_{1}}\right) \cdots\left(1+\overline{\pi_{r}}\right)=\sum_{\mu \leq b} \overline{\pi_{\mu}}$ est une expression dont le développement ne fait apparaître aucun carré $\bar{\pi}_{i}^{2}$. Les éléments de l'intervalle $[\leq b]$ sont en correspondance bijective avec les sous-mots de la décomposition réduite $b=\sigma_{1} \sigma_{2} \cdots \sigma_{r}$. Cet intervalle est booléen et l'on a $G(r, 1)=(1+z)^{r}$, ce qui résulte aussi du fait bien connu que l'idéal d'un cycle $b$ est un intervalle booléen.

Comme exemple de la proposition, on a

$G(7,4)=\left(1+z+z^{2}+z^{3}+z^{4}\right) G(6,4)+z^{7}\left(3+2 z+z^{2}\right) G(6,3)+z^{14}(3+$ z) $G(6,2)+z^{21} G(6,1)=1+10 z+54 z^{2}+209 z^{3}+\ldots+252 z^{26}+28 z^{27}+z^{28}$ est le polynôme de Poincaré d'un pavé de cardinal 1315666 de $\mathfrak{S}(11)$ que nous nous dispenserons d'énumérer.

Table des coefficients des $G(r, p)$, pour $4 \geq r \geq p \geq 2$

$\begin{array}{ccccccccccccccc}G(2,2) & 1 & 3 & 5 & 4 & 1 & & & & & & & & & 14 \\ G(3,2) & 1 & 4 & 9 & 13 & 12 & 6 & 1 & & & & & & \\ G(3,3) & 1 & 5 & 14 & 29 & 45 & 53 & 46 & 27 & 9 & 1 & & & \\ G(4,2) & 1 & 5 & 14 & 26 & 35 & 34 & 22 & 8 & 1 & & & & \\ G(4,3) & 1 & 6 & 20 & 49 & 95 & 150 & 195 & 206 & 173 & 110 & 48 & 12 & 1 & 1066 \\ G(4,4) & 1 & 7 & 27 & 76 & 174 & 337 & 562 & 815 & 1029 & 1125 & 1052 & 823 & 521 & \\ & & & & & & & & & & 252 & 84 & 16 & 1 & 6902\end{array}$

7. Groupe $B_{n}$. - Le goupe $B_{n}$ est engendré par $n$ générateurs $s_{1}, \ldots, s_{n}$ qui satisfont aux mêmes relations que les générateurs du groupe symétrique, mis à part le remplacement de $\left(s_{1} s_{2}\right)^{3}=1$ par

$$
\left(s_{1} s_{2}\right)^{4}=1
$$


Chaque élément peut être décrit par sa décomposition réduite lexicographiquement minimale ( $d r l m)$. Ecrivant $i$ pour $s_{i}$, celle-ci est un sous-mot de la décomposition de l'élément maximal :

$$
\text { (1) }(212)(32123) \cdots(n \ldots 212 \ldots n)
$$

qui est formé de facteurs gauches de chacun des blocs indiqués entre parenthèses.

On peut donc coder bijectivement un élément de $B_{n}$ par la suite des longueurs des facteurs successifs de sa $d r l m$; les éléments de $B_{n}$ sont en bijection avec les vecteurs $c$ dans $\mathbb{N}^{n}, c \leq[1,3,5, \ldots, 2 n+1]$ que nous appelerons codes.

Les éléments de $B_{n}$ peuvent aussi être représentés par les permutations signées de $\{1, \ldots, n\}$, en interprétant $s_{1}$ comme le morphisme de $\mathbb{Z}^{n}$ :

$$
u=\left[u_{1}, \ldots, u_{n}\right] \rightarrow u s_{1}=\left[-u_{1}, u_{2}, \ldots, u_{n}\right]
$$

et $s_{i}, i=2, \ldots, n$, comme la transposition simple échangeant les composantes $u_{i-1}$ et $u_{i}$.

Une deuxième représentation est le plongement dans $\mathfrak{S}(2 n)$ défini par

$$
s_{1} \rightarrow \sigma_{n}, s_{2} \rightarrow \sigma_{n-1} \sigma_{n+1}, \ldots, s_{n} \rightarrow \sigma_{1} \sigma_{2 n-1}
$$

où les $\sigma_{i}$ sont les transpositions simples de $\mathfrak{S}(2 n)$. L'image de $B_{n}$ est alors l'ensemble des permutations $\mu$ palindromes, i.e. telles que $\mu_{i}+\mu_{2 n-i}=$ $2 n+1, i=1, \ldots, n$.

Le passage du code à chacune de ces deux représentations est immédiat et correspond à compter les inversions. Ainsi le code $[1,0,5,2]$ correspond à la décomposition réduite $(1)()(32123)(43)$, à la permutation signée $[-1,4,2,-3]$ et au palindrome 73154862 . En effet, $c_{4}=2$ indique la place de 4 (à partir de la droite) dans la permutation signée et la place de 8 dans le palindrome; $c_{3}=5$ donne la place de -3 dans $[-1,2,-3]$, le signe - impliquant que l'on compte la place à partir de la gauche en ajoutant l'ordre $(=3)$, ainsi que la place de 7 dans le palindrome 735462.

On passe de la permutation signéee au palindrome par duplication et retour à l'alphabet $\{1,2, \ldots, 2 n\}$ par translation de $n$ :

$$
[-1,4,2,-3] \rightarrow[3,-2,-4,1], \quad[-1,4,2,-3] \sim 73154862
$$

Le générateur $s_{1}$ joue un rôle particulier, ce qui conduit à distinguer deux types de facteurs $v$ d'une $d r l m$ : 
- colonne si $s_{1} \notin v$ (i.e. $v=s_{k} \cdots s_{k-i}, i<k-1$ )

- équerre sinon.

Le produit d'une colonne ou d'une équerre par un générateur $s_{i}$ est instantané et permet ainsi de d'expliciter l'action, à gauche ou à droite, de $B_{n}$ sur les codes.

Soit un élément de $B_{n}$ dont le code n'a que deux composantes non nulles. Cet élément n'est une bigrassmannienne que dans les seuls cas suivants:

(cc) $c_{1}=0=c_{k-1} ; c_{k}=c_{k+1}<k ; c_{k+2}=0=\cdots=c_{n}$

(ee) $c_{1}=0=c_{k-1} ;=c_{k+1}=c_{k}+1 \geq k+1<k ; c_{k+2}=0=\cdots=c_{n}$

(ec) $c_{1}=0=c_{k-1} ; c_{k} \geq k, c_{k+1}=2 k+1-c_{k} ; c_{k+2}=0=\cdots=c_{n}$

Les facteurs correspondants de la $d r l m$ sont respectivement (colonne, colonne); (équerre, équerre); (équerre, colonne).

Par exemple, pour $k=5$, avec $i$ au lieu de $s_{i}$, les $d r l m$ suivantes illustrent ces trois types:

(cc) (543) (654)

(ee) (5432123) (65432123)

(ec) (5432123) (654)

Par récurrence sur le nombre de facteurs, on en déduit la caractérisation des codes des bigrassmanniennes de $B_{n}$ :

LEMME 7.1. - Un vecteur c dans $\mathbb{N}^{n}$ est le code d'une bigrassmannienne de $B_{n}$ ssi

i) $c \leq[1,3, \ldots, 2 n+1]$; ses composantes non nulles sont consécutives et constituent une suite d'équerres suivie par une suite de colonnes (l'une de ces deux suites peut être vide)

ii) chaque paire de composantes adjacentes non nulles vérifie la condition cc), ee) ou ec) indiquée par son type.

Par exemple, l'ensemble des codes des bigrassmanniennes de $B_{3}$ est $\{[0,3,4] ;[1,2,3] ;[0,0,5],[0,2,3],[1,2,2] ;[0,0,4],[0,3,1],[0,2,2]$; $[0,0,3],[0,3,0],[1,2,0],[1,1,1] ;[0,0,2],[0,1,1],[0,2,0],[1,1,0]$; $[0,0,1],[0,1,0],[1,0,0]\}$

Du lemme précédent, on déduit la fonction génératrice du nombre des bigrassmanniennes :

Lemme 7.2. - La fonction $\sum_{n=1}^{\infty} z^{n-1} \operatorname{card}\left(\left\{\operatorname{bigr}\left(B_{n}\right)\right\}\right)$ est égale à

$$
(1-z)^{-5}+z(1-z)^{-4}=1+6 z+19 z^{2}+45 z^{3}+90 z^{4}+\ldots
$$

Nous allons voir que l'ensemble des bigrassmanniennes contient strictement la base de $B_{n}$, pour $n \geq 4$. 
Comme l'ordre de Bruhat sur $B_{n}$ coïncide avec la restriction de l'ordre de Bruhat sur $\mathfrak{S}(2 n)$ au sous-ensemble des permutations palindromes [Lak], [Pr], on peut décrire l'ordre de $B_{n}$ en utilisant les bigrassamnniennes de $\mathfrak{S}(2 n)$.

Tout d'abord, on remarque que pour les permutations palindromes

$$
\mu \geq \operatorname{bigr}\left[r_{0}, r_{1}, r_{2}, r_{3}\right] \Leftrightarrow \mu \geq \operatorname{bigr}\left[r_{3}, r_{2}, r_{1}, r_{0}\right] .
$$

Appelons couple miroir un tel couple de bigrassmanniennes, invariant par le retournement $\left[r_{0}, r_{1}, r_{2}, r_{3}\right] \rightarrow\left[r_{3}, r_{2}, r_{1}, r_{0}\right]$.

En particulier, l'ensemble des rectrices d'une permutation palindrome est formé de couples miroirs dont il suffit de se donner un seul des deux éléments.

Définition. - Les rectrices d'un élément de $B_{n}$ sont les rectrices $\left[r_{0}, r_{1}, r_{2}, r_{3}\right]$ de son image palindrome qui sont telles que $\left\{r_{0}<r_{3}\right\}$ ou $\left\{r_{0}=r_{3} \& r_{1} \leq r_{2}\right\}$.

Les éléments de $B_{n}$ sont déterminés par leurs rectrices, puisque les éléments de $\mathfrak{S}(2 n)$ le sont. L'ordre de Bruhat sur les éléments de $B_{n}$ est induit par l'ordre sur les bigrassmanniennes de $\mathfrak{S}(2 n)$. Plus explicitement, tenant compte de ce que l'on n'a pris que la moitié des rectrices des palindromes par raison de symétrie, on a

$$
\left[r_{0}, r_{1}, r_{2}, r_{3}\right] \leq_{B_{n}}\left[p_{0}, p_{1}, p_{2}, p_{3}\right]
$$

ssi

$$
\left[r_{0}, r_{1}, r_{2}, r_{3}\right] \leq_{\mathfrak{S}(2 n)}\left[p_{0}, p_{1}, p_{2}, p_{3}\right] \text { ou }\left[r_{0}, r_{1}, r_{2}, r_{3}\right] \leq_{\mathfrak{S}(2 n)}\left[p_{3}, p_{2}, p_{1}, p_{0}\right]
$$

LEMme 7.3. - Soit $b=\left[r_{0}, r_{1}, r_{2}, r_{3}\right]$ une bigrassmannienne de $\mathfrak{S}(2 n)$. Alors l'ensemble des permutations palindromes $\geq b$ admet un élément inférieur, noté $b_{+}$. Symétriquement, l'ensemble des permutations palindromes inférieures à une cobigrassmannienne c de $\mathfrak{S}(2 n)$ a un supremum, noté $c_{-}$.

De plus l'élément correspondant à $b_{+}\left(\begin{array}{rrr}\text { resp } & c_{-}\end{array}\right)$dans $B_{n}$ est une bigrassmannienne (resp. cobigrassmannienne).

Preuve. - On peut supposer que $b=\left[r_{0}, r_{1}, r_{2}, r_{3}\right]$ avec $r_{0}<r_{3}$ ou $r_{0}=r_{3} \& r_{1} \leq r_{2}$. On a quatre cas possibles, suivant que $r_{0}+r_{1} \leq n$ ou non, et $r_{0}+r_{2} \leq n$ ou non.

Prenons par exemple $r_{0}+r_{1}>n$ et $r_{0}+r_{2} \leq n$. On cherche les palindromes tels que le facteur gauche de longueur $r_{0}+r_{1}$ contienne au moins $r_{1}$ valeurs $>r_{0}+r_{2}$. L'ensemble des valeurs $\{1, \ldots, 2 n\}$ est coupé en trois intervalles $X=\left\{1, \ldots, r_{0}+r_{2}\right\}, Y^{\prime}=\left\{r_{0}+r_{2}+1, \ldots, r_{1}+r_{3}\right\}$, $Y^{\prime \prime}=\left\{r_{1}+r_{3}+1, \ldots, 2 n\right\}$. 
De même, les permutations, considérées comme mots, sont coupées en trois segments de longueurs respectives $r_{2}+r_{3}, r_{0}+r_{1}-r_{2}-r_{3}, r_{2}+r_{3}$. Pour la projection $p: X \rightarrow x, Y^{\prime} \rightarrow y^{\prime}, Y^{\prime \prime}$ sur l'alphabet $\left\{x<y^{\prime}<y^{\prime \prime}\right\}$, on a que $\mu \geq b$ implique que le facteur gauche de longueur $r_{0}+r_{1}$ contient au moins $r_{1}$ valeurs dans $Y^{\prime} \cup Y^{\prime \prime}$. Tenant compte de ce que $\mu$ est palindrome, cela implique que

$$
\mu \geq\left[r_{0}, r_{1}, r_{2}, r_{3}\right] \Leftrightarrow p(\mu) \geq v:=x^{r_{0}} y^{\prime \alpha} y^{\prime \prime r_{2}} y^{\prime \beta} x^{r_{2}} y^{\prime \alpha} y^{\prime \prime r_{0}}
$$

avec $\alpha=r_{3}-r_{0}, \beta=r_{0}+r_{1}-n$.

Ce mot est l'inf des palindromes d'évaluation $x^{r_{0}+r_{2}} y^{\prime r_{1}+r_{3}-r_{0}-r_{2}} y^{\prime \prime r_{0}+r_{2}}$ qui soient plus grands que $p(b)$. Le palindrome minimum qui se projette sur $v$ est, en marquant les mêmes coupures que $v$, $b_{+}:=1 \ldots r_{0}\left|r_{0}+r_{2}+1 \ldots, r_{2}+r_{3}\right| r_{1}+r_{3}+1 \ldots r_{1}+r_{2}+r_{3} \mid$ $r_{2}+r_{3}+1 \ldots r_{0}+r_{1}\left|r_{0} \ldots r_{0}+r_{2}\right| r_{0}+r_{1}+1 \ldots r_{1}+r_{3} \mid 2 n-r_{0}+1 \ldots 2 n$.

Comme dans le cas du groupe symétrique, on termine l'analyse en vérifiant que $p\left(b_{+}\right)$reste bien minimum lorsque l'on fait varier la projection d'alphabet $p:\{1 \ldots 2 n\} \rightarrow\{x, y\}$.

Les trois autres cas se traitent explicitement de la même manière.

Etant donnée une bigrassmannienne $b=\left[r_{0}, r_{1}, r_{2}, r_{3}\right]$ de $\mathfrak{S}(2 n)$, la cobigrassmannienne qui clive avec elle est $c=\omega b^{\prime}$, avec $b^{\prime}=\left[r_{1}-1, r_{0}+\right.$ $\left.1, r_{3}+1, r_{2}-1\right]$. De l'existence de $b^{\prime}{ }_{+}$, on déduit donc par multiplication par $\omega$ l'existence d'une permutation palindrome maximale dans l'idéal $[\leq c]$ complémentaire de $[b \leq]$, qui n'est autre que $\omega b^{\prime}{ }_{+}$.

Enfin, on voit, sur son expression explicite, que $b_{+}$a deux descentes, lesquelles sont à des places symétriques, de même que son inverse. En d'autres termes, l'élément correspondant de $B_{n}$ est une bigrassmannienne

ThÉORÈme 7.4. - L'image de la base de $B_{n}$ dans $\mathfrak{S}(2 n)$ est l'ensemble des palindromes $\left\{b_{+}\right\}$qui sont les Sup respectifs des couples miroirs de bigrassmanniennes de $\mathfrak{S}(2 n)$, et en correspondance bijective avec ces derniers.

Le treillis enveloppant de $B_{n}$ est distributif, i.e. chaque élément de la base clive $B_{n}$ en une union disjointe de deux intervalles.

La base est un sous-ensemble strict de l'ensemble des bigrassmanniennes de $B_{n}$.

Remarque. - Le cardinal de la base de $B_{n}$ est $n\left(2 n^{2}+1\right) / 3$, et donc la fonction génératrice des cardinaux est

$$
(1+z)^{2} /(1-z)^{4}=1+z+19 z^{2}+44 z^{3}+85 z^{4}+\cdots
$$


Le rapport $\operatorname{card}\left(\operatorname{base}\left(B_{n}\right)\right) / \operatorname{card}\left(\operatorname{Bigr}\left(B_{n}\right)\right)$ tend vers 0 comme $1 / n$ lorsque $n$ tend vers l'infini.

La seule bigrassmannienne de $B_{4}$ qui n'appartient pas à la base a pour code [0042] et $d r l m s_{3} s_{2} s_{1} s_{2} s_{4} s_{3}$; elle est le sup des deux éléments de la base [0050] $=s_{3} s_{2} s_{1} s_{2} s_{3}$ et [0022] $=s_{3} s_{2} s_{4} s_{3}$. Les cinq bigrassmanniennes de $B_{5}$ exclues de la base ont pour codes respectifs

$$
\text { [00053], [00420], [00062], [00422], [00453] }
$$

8. Annexe. - Commençons par rappeler brièvement la preuve classique du théorème de Mac Neille fournie par la méthode de O.Ore.

Soit donnée une relation $\mathcal{R} \subset \bar{X} \times X$ entre deux ensembles $\bar{X}$ et $X$. Pour chaque $x \in X$, nous posons $x \bar{\rho}=\{\bar{x} \in \bar{X}:(\bar{x}, x) \in \mathcal{R}\}$ et $Y \bar{\rho}=\cap\{y \bar{\rho}: y \in Y\}$ pour chaque partie $Y \neq \emptyset, X$ de $X$. On convient de plus que $\emptyset \bar{\rho}=\bar{X}$ et $X \bar{\rho}=\emptyset$.

On définit de même $\rho$ en échangeant le rôle de $X$ et de $\bar{X}$, et tous les énoncés qui suivent restent valides par cet échange, un phénomène que nous nous dispenserons souvent de mentionner.

Il est clair que $\bar{\rho}: 2^{X} \rightarrow 2^{\bar{X}}$ est décroissante

$$
\left(Y^{\prime} \subset Y \in 2^{X} \Rightarrow Y \bar{\rho} \subset Y^{\prime} \bar{\rho}\right)
$$

et que $\bar{\rho} \rho: 2^{X} \rightarrow 2^{X}$ est croissante $\left(Y \in 2^{X} \Rightarrow Y \subset Y \bar{\rho} \rho\right)$.

On a donc $\bar{\rho} \rho \bar{\rho}=\bar{\rho}($ et $\rho \bar{\rho} \rho=\rho)$ puisque

$$
Y \bar{\rho} \subset(Y \bar{\rho}) \rho \bar{\rho}=Y \bar{\rho} \rho \bar{\rho}=(Y \bar{\rho} \rho) \bar{\rho} \subset Y \bar{\rho}
$$

identiquement.

Soit maintenant $\bar{T}=\left\{Y \bar{\rho}: Y \in 2^{X}\right\} \in 2^{2^{\bar{X}}}$. D'après la définition de $\bar{\rho}: 2^{X} \rightarrow 2^{\bar{X}}$, la famille $(\bar{T}, \subset)$ de parties de $\bar{X}$ munie de l'ordre d'inclusion, est un treillis dans lequel l'opération Inf est l'intersection ensembliste $\cap$. Plus précisément, $(\bar{T}, \subset)$ est le plus petit treillis contenant tous les $x \bar{\rho}$ $(x \in X)$.

Symétriquement, $T=\left\{\bar{Y} \rho: \bar{Y} \in 2^{\bar{X}}\right\} \in 2^{2^{X}}$ est le treillis engendré par les $\bar{x} \rho(\bar{x} \in \bar{X})$. Si $Y \in T$, on a, par définition, $Y \bar{\rho} \in \bar{T}$ et $Y=\bar{Y}^{\prime} \rho$ pour une certaine partie $\bar{Y}^{\prime}$ de $\bar{X}$. On a donc

$$
Y \bar{\rho} \rho=\bar{Y}^{\prime} \rho \bar{\rho} \rho=\bar{Y}^{\prime} \rho=Y,
$$

ce qui montre que les restrictions $\bar{\rho}: T \rightarrow \bar{T}$ et $\rho: \bar{T} \rightarrow T$ sont deux antiisomorphismes (de la relation d'ordre $\subset$ ) inverses l'un de l'autre. 
Autrement dit, si $\left\{Y_{i}: i \in \mathcal{J}\right\}$ est une famille d'éléments de $T$, on a $\operatorname{Inf}\left\{Y_{i}: i \in \mathcal{J}\right\}=\cap\left\{Y_{i}: i \in \mathcal{J}\right\} \in T$, cependant que $\operatorname{Sup}\left\{Y_{i}: i \in \mathcal{J}\right\}=$ $\bar{Z} \rho$, avec $\bar{Z}=\cap\left\{Y_{i} \bar{\rho}: i \in \mathcal{J}\right\} \in \bar{T}$.

Nous appelerons $T$ et $\bar{T}$ le $X$-treillis et le $\bar{X}$-treillis engendrés par $\mathcal{R}$ et nous utiliserons l'abréviation

$$
\operatorname{Diag}(\mathcal{R})=\left\{(\bar{Y}, Y) \in 2^{\bar{X}} \times 2^{X}: \bar{Y} \rho=Y ; Y \bar{\rho}=\bar{Y}\right\} \subset \bar{T} \times T
$$

En considérant, comme ci-dessus, $\mathcal{R}$ comme une matrice booléenne, une paire de parties $(\bar{Y}, Y)$ sera appelé un rectangle (de 1 ) ssi $\bar{Y} \times Y$ est contenu dans $\mathcal{R}$. Par construction, les paires $(\bar{Y} \times Y) \in \operatorname{Diag}(\mathcal{R})$ sont les rectangles maximaux de $\mathcal{R}$, en ce sens que, étant donné $Y \subset X, \bar{Y}$ est la plus grande partie de $\bar{X}$ telle que $\bar{Y} \times Y$ soit un rectangle, et que l'on ait la condition symétrique sur $Y$ par rapport à $\bar{Y}$. Par définition, les éléments de $\bar{T}$ et $T$ sont les projections de $\operatorname{Diag}(\mathcal{R})$ sur $2^{\bar{X}}$ et $2^{X}$.

Considérons deux parties $\bar{X}_{1}$ de $\bar{X}$ et $X_{2}$ de $X$.

Elles définissent par restriction trois autres relations

$$
\mathcal{R}_{1}=\mathcal{R} \cap\left(\bar{X}_{1} \times X\right) ; \mathcal{R}_{2}=\mathcal{R} \cap\left(\bar{X} \times X_{2}\right) ; \mathcal{R}_{3}=\mathcal{R} \cap\left(\bar{X}_{1} \times X_{2}\right)
$$

engendrant les paires de treillis $\left(\bar{T}_{1}, T_{1}\right),\left(\bar{T}_{2}, T_{2}\right),\left(\bar{T}_{3}, T_{3}\right)$.

Par définition $T_{1}$ (resp. $\bar{T}_{2}$ ) est une famille de parties de $X$ (resp. $\bar{X}$ ) contenue dans $T$ (resp. dans $\bar{T}$ ) et l'injection naturelle $T_{1} \rightarrow T$ (resp. $\bar{T}_{2} \rightarrow \bar{T}$ ) est un morphisme pour l'opération Inf puisque cette dernière n'est autre que l'opération ensembliste $\cap$.

On a de même des morphismes canoniques $\bar{T}_{3} \rightarrow \bar{T}_{1}$ et $T_{3} \rightarrow T_{2}$.

Lemme 8.1. - Si $\bar{X}_{1}$ et $X_{2}$ sont tels que $T_{1}=T$ et $\bar{T}_{2}=\bar{T}$, on a

$$
T_{3}=T \quad \text { et } \quad \bar{T}_{3}=\bar{T}
$$

Preuve. - En posant $X_{1}=X, \bar{X}_{2}=\bar{X}, \bar{X}_{3}=\bar{X}_{1}$ et $X_{3}=X_{2}$, les hypothèses et la conclusion peuvent s'écrire

$$
\operatorname{Diag}(\mathcal{R})=\operatorname{Diag}(\mathcal{R}) \cap\left\{2^{\overline{X_{i}}} \times 2^{X_{i}}\right\}\left(=\operatorname{Diag}\left(\mathcal{R}_{i}\right)\right)
$$

respectivement pour $i=1,2$ et pour $i=3$.

Supposons que $(\bar{Y}, Y)$ soit un rectangle maximal dans $\bar{X}_{2} \times X_{1}$. On a d'une part $\bar{Y} \times Y \subset \mathcal{R}_{3}$, donc $\subset \mathcal{R}$, et d'autre part que pour chaque $z \in X_{1} \backslash Y$, il y au moins un $\bar{y} \in \bar{Y}$ pour lequel $(y, z) \notin \mathcal{R}_{3}$, donc $\notin \mathcal{R}$, et symétriquement pour chaque $z \in \bar{X}_{2} \backslash \bar{Y}$. Ceci implique que $(\bar{Y}, Y)$ est la 
restriction à $X_{2} \times Y$ du rectangle maximal $(Y \bar{\rho}, \bar{Y} \rho) \in \operatorname{Diag}(\mathcal{R})$ et établit que

$$
\operatorname{Diag}\left(\mathcal{R}_{3}\right) \subset \operatorname{Diag}(\mathcal{R}) \cap\left(2^{\overline{X_{1}}} \times 2^{X_{2}}\right) .
$$

Réciproquement, soit maintenant $\bar{Y} \times Y \in \operatorname{Diag}(\mathcal{R})$. Sa restriction à $\bar{X}_{1} \times X_{2}$ est encore un rectangle dont il suffit de prouver qu'il est maximal pour chaque $z \in X \backslash Y$. Or la maximalité de $\bar{Y} \times Y$ implique l'existence d'au moins un $\bar{y} \in \bar{Y}$ tel que $(\bar{y}, z) \notin \mathcal{R}$ et l'hypothèse $\operatorname{Diag}\left(\mathcal{R}_{1}\right)=\operatorname{Diag}(\mathcal{R})$ implique que l'on puisse prendre un tel $\bar{y}$ dans $\bar{Y} \cap \bar{X}_{1}$.

Un argument symétrique s'applique aux $\bar{z} \in \bar{X} \backslash \bar{Y}$, ce qui achève la preuve

Nous en venons maintenant à la preuve des résultats énoncés au paragraphe 2 .

Lemme 2.3. - Soit $(X, \leq)$ un ensemble ordonné. Un élément $x \neq \wedge X$ appartient à la base $B$ ssi il est un élément minimal de $X \backslash[\leq z]$ pour au moins un $z \in X$.

Quand $(X, \leq)$ est un treillis, cette condition est remplie ssi $x$ couvre exactement un autre élément de $X$.

Preuve. - Soit $x$ un élément de $X \backslash \wedge X$. Par définition, il appartient au complément de $X \backslash B$ de la base ssi il existe une partie $Y \neq \emptyset$ de $X$ telle que

$$
x \notin Y \quad \& \quad[x \leq]=\cap\{[y \leq]: y \in Y\} .
$$

On observe d'abord que cette relation est vraie pour un $Y \neq \emptyset$ ssi elle est vraie pour le cas particulier de $Y=[<x](:=[x \leq] \backslash x)$. En effet, si $Y$ satisfait $(\star)$ on a $y \in[<x]$ pour chaque $y \in Y$, et d'autre part $Y \cup\{y\}$ satisfait encore $(\star)$ pour chaque $y \in[<x]$. On a donc $x \in X \backslash B$ ssi

$$
[x \leq]=Z \text {, où } \quad Z:==\cap\{[y \leq]: y \in[<x]\} .
$$

Par construction $Z \backslash[\leq x]$ est l'ensemble des $z \in X$ tel que $x$ soit un élément minimal de $X \backslash[\leq z]$. On a donc $x \in X \backslash B$ ou $x \in B$ selon que $Z \backslash[\leq x]$ est vide ou non. Il est clair que $x \in B$ quand $x(\neq \wedge X)$ est un élément minimal de $X$, car il suffit de prendre n'importe quel $z \in X \backslash[\leq x]$. Il en est de même quand $x$ couvre un seul élément, qui est alors $\operatorname{Sup}([<x])$. Le cas particulier où $(X, \leq)$ est un treillis est trivial puisque l'on a alors $\wedge X \neq \emptyset$ et pour tout $x \in X \backslash \wedge X, \operatorname{Sup}([<x] \neq \emptyset)$

Proposition 2.4. - La projection sur la base $\beta: X \ni x \rightarrow B \cap[\leq x] \in$ $\left(2^{B}, \subset\right)$ est un isomorphisme d'ordre et l'on a $B \subset C$ pour toute partie $C$ de $X$ ayant la même propriété. 
Preuve. — Soient $y, z \in X$. Si $Y:=[\leq y] \backslash[<z]$ est vide, c'est-à-dire si $y \leq z$ on a $y \beta \subset z \beta$. Pour établir que $\beta$ est isomorphisme il suffit donc de montrer que $Y$ contient au moins un élément de la base quand $Y \neq \emptyset$.

Or comme $Y$ est fini, il contient au moins un élément minimal $b$. On a $b \neq \wedge X$ puisque $\wedge X \in[\leq z]$ quand $\wedge X \neq \emptyset$, et donc $b \in B$ d'après le lemme 2.3 .

Soit maintenant $C$ une partie de $X$ telle que l'application $\gamma: X \ni$ $x \longrightarrow x \gamma=C \cap[\leq x]$ soit un isomorphisme. Supposons qu'il existe un $b \in B \backslash C$. D'après le lemme 2.3 il existe au moins un $z \in X \backslash[b \leq]$ tel que $[<b] \subset[\leq z]$. On a alors $b \gamma=C \cap[<b] \subset z \gamma=C \cap[\leq z]$, contredisant l'hypothèse que $\gamma$ est un isomorphisme. Donc $C$ contient $B$.

On définit de même l'application $\bar{\beta}: X \in x \longrightarrow \bar{B} \cap[x \leq]$, et l'on a pour $\bar{\beta}$ des énoncés symétriques de ceux pour $\beta$. On notera que $x \beta=\emptyset$ ssi $x=\wedge X$, et $x \beta=X$ ssi $x=\vee X$.

Exemples. - Soit $X=\{1,2,3,4\}$ avec la relation d'ordre $\leq$ définie par $\{1,2,3\}=[\leq 3]$ et $\{1,2,4\}=[\leq 4]$. Tous les éléments de $X$ appartiennent à la base et à la cobase. La projection $\gamma \operatorname{sur} C=\{1,2,3\}$ est injective, mais elle n'est pas un isomorphisme puisque $4 \gamma=\{1,2\} \subset 3 \gamma=\{1,2,3\}$ bien que 3 et 4 soient incomparables. Le treillis enveloppant de $X$ est obtenu en ajoutant à $X$ un plus petit et un plus grand élément, et aussi un élément $t$ tel que

$$
t=\operatorname{Sup}(\{1,2\})=\operatorname{Inf}(\{3,4\}) .
$$

Ce treillis est le treillis enveloppant de $\mathfrak{S}(3)$ (muni de l'ordre fort) vu au 5 .

La proposition 2.4 requiert une condition topologique quand $X$ n'est pas fini. Ainsi elle n'est pas vraie quand $(X, \leq)$ est un intervalle de la droite réelle $\mathbb{R}$ puisque dans ce cas la base et la cobase sont des ensembles vides. Par contre elle est vraie pour $(\mathbb{Z}, \leq)$ qui est égal à sa base, puisque la condition des chaînes descendantes est vérifiée pour toute partie $[\leq y] \backslash[\leq z]$ non vide. Le treillis enveloppant est obtenu en ajoutant à $\mathbb{Z}$ un plus petit et un plus grand élément.

La proposition 2.4 est aussi vérifiée par le treillis distributif $\left(2^{\mathbb{Z}}, \subset\right)$ dont les éléments sont les parties de $\mathbb{Z}$.

Enfin soit $X$ l'ensemble union de l'intervalle $[0,1]$ de $\mathbb{R}$, muni de son ordre naturel et d'un ensemble $B=\left\{b_{x}: x \in[0,1]\right\}$, d'éléments incomparables. On ajoute les relations croisées $0<b_{x}$ et $b_{x}<x^{\prime}$ ssi $x \leq x^{\prime} \in[0,1]$. Alors $B$ est à la fois la base et la cobase de $X$. L'application $\beta: X \longrightarrow B$ est un isomorphisme d'ordre, ce qui n'est pas le cas de $\bar{\beta}: X \longrightarrow B$.

C'est un problème ouvert de trouver des catégories non triviales de treillis pour lesquelles la proposition 2.4 et sa symétrique sont vraies. 
Soit $\mathcal{R}^{\prime} \subset \bar{B} \times B$ la relation définie par $(\bar{b}, b) \in \mathcal{R}^{\prime}$ ssi $\bar{b} \geq b$, c'est-à-dire, de façon équivalente, ssi $b \in \bar{b} \beta$ ou $\bar{b} \in b \bar{\beta}$.

Proposition 2.5. - La relation $\mathcal{R}^{\prime}$ est une relation basique.

Preuve. - Comme $X \backslash[\leq x] \neq \emptyset$, sauf si $x=\wedge X$, la preuve précédente montre que $\bar{b} \beta \neq B$ pour chaque $\bar{b} \in \bar{B}$. Supposons qu'un élément $\bar{b} \in \bar{B}$ et une partie $\bar{C}$ de $\bar{B}$ soient tels que $\bar{b} \beta=\cap\{\bar{c} \beta: \bar{c} \in \bar{C}\}$. Comme $\beta$ est un isomorphisme d'après (2.4), on a $\bar{c} \in[\bar{b} \leq]$ pour tout $\bar{c} \in \bar{C}$, et réciproquement $\bar{b} \beta \subset x \beta$ pour tout $x \in[\bar{b} \leq]$; l'hypothèse implique que $\bar{b} \beta=\cap\{x \beta: x \in[\bar{b} \leq]\}$ et enfin $\bar{b} \in \bar{C}$ d'après la définition de $\bar{B}$.

Autrement dit, aucune ligne de $\mathcal{R}^{\prime}$ n'est l'intersection d'autres lignes. Ceci achève la preuve par symétrie entre $B$ et $\bar{B}$

Nous appelerons désormais la relation $\mathcal{R}^{\prime}$ précédente la relation basique de l'ensemble ordonné $(X, \leq)$.

On voit facilement qu'un élément $x \in X$ appartient à $\bar{B} \cap B$ ssi $x \bar{\beta} \times x \beta$ est un rectangle de $\mathbf{1}$ (au sens donné plus haut à ce terme) dans la relation basique.

Donnons maintenant à la preuve du théorème 2.6.

Prenant une copie $\bar{X}$ de $X$ et une bijection $X \ni x \rightarrow \bar{x} \in \bar{X}$, nous associons à $(X, \leq)$ sa matrice d'incidence, c'est-à-dire la relation $\mathcal{R}$ dans $\bar{X} \times X$ telle que $\left(\bar{x}_{1}, x_{2}\right) \in \mathcal{R}$ ssi $x_{1} \geq x_{2}$. La relation basique de $(X, \leq)$ est simplement la restriction de $\mathcal{R}$ à $\bar{B} \times B$.

Utilisant les conventions du début de cette annexe, on voit que pour chaque $x \in X$, on peut identifier la colonne $x \bar{\rho}$ à l'intervalle supérieur $[x \leq]$ et la ligne $\bar{x} \rho$ à $[\leq \bar{x}]$. Le treillis $T$ et le treillis antiisomorphe $\bar{T}$ de la construction de Ore sont donc respectivement le treillis enveloppant de $(X, \leq)$ et celui de l'ensemble antiisomorphe $(\bar{X}, \geq)$.

Comme les applications $\beta$ et $\bar{\beta}$ sont injectives, les relations $\mathcal{R} \cap(\bar{X} \times B)$ et $\mathcal{R} \cap(\bar{B} \times X)$ engendrent des paires de treillis isomorphes à $\bar{T} \times T$. Les hypothèses du lemme 2.8 sont donc satisfaites, ce qui permet de conclure que la relation basique $\mathcal{R} \cap(\bar{B} \times B)$ de $(X, \leq)$ est aussi celle du treillis $T$ $\square$

Nous en venons maintenant au clivage.

Soit $\mathcal{R} \subset \bar{B} \times B$ la relation basique d'une relation d'ordre $(X, \leq)$.

Le lemme 2.2 montre que deux éléments $\bar{x}, x \in X$ sont tels que $X$ n'est l'union disjointe de $[\leq \bar{x}]$ et $[x \leq]$ que si $(\bar{x}, x) \in \bar{B} \times B$. En ce cas, $x$ détermine $\bar{x}$ et réciproquement.

Il est clair que si $\left(\bar{b}_{1}, b_{1}\right)$ et $\left(\bar{b}_{2}, b_{2}\right)$ sont deux paires clivantes, on a $b_{1} \leq b_{2}$ ssi $\bar{b}_{1} \leq \bar{b}_{2}$. Ceci conduit à définir le morphisme partiel $N E G$ de $B$ dans $\bar{B}$ en posant : 


$$
\begin{aligned}
N E G(b) & =\bar{b} \text { s'il existe } \bar{b} \in \bar{B} \text { tel que }(\bar{b}, b) \text { soit un clivage } \\
& =\emptyset \text { sinon }
\end{aligned}
$$

Nous supposons désormais que $X$ est le treillis défini par $R$.

Soient $b \in B, Y:=X \backslash[b \leq]$ et $s:=\operatorname{Sup}(Y)$. L'ensemble $X$ est l'union de $[\leq s]$ et de $[b \leq]$, cette union étant disjointe ssi $b \notin[\leq s]$. Donc $N E G(b)=s$ ou $\emptyset$ selon que $b \notin[\leq s]$ ou non.

Considérons alors l'unique élément $b_{-}$couvert par $b$. Comme $b_{-} \in Y$, on a $\operatorname{Inf}\left(b_{-}, s\right) \in\left\{b, b_{-}\right\}$et d'autre part $\operatorname{Sup}(\operatorname{Inf}(b, y): y \in Y)=b_{-}$.

Par conséquent la relation de distributivité

$$
\operatorname{Inf}\left(b_{-}, \operatorname{Sup}(Y)\right)=\operatorname{Sup}\left(\left\{\operatorname{Inf}\left(b_{-}, y\right): y \in Y\right\}\right)
$$

implique $b \notin[\leq s]$ et enfin $N E G(b)=s \neq \emptyset$.

Compte tenu de la symétrie entre $B$ et $\bar{B}$, cette remarque établit la partie directe du théorème 2.8 .

Revenant au cas général, nous isolons le

Lemme 8.5. - Pour chaque $b \in B$, on a $N E G(b) \neq \emptyset$ ssi il existe un $\bar{b} \in \bar{B}$ tel que $\bar{b} \beta=B \backslash[b \leq]$.

Preuve. - Il suffit d'observer qu'avec les notations introduites plus haut, on a $b \notin[\leq s]$ ssi $s \beta=Y \cap B$

Comme $B \cap[b \leq]$ est l'ensemble des $b^{\prime} \in B$ tels que $b^{\prime} \bar{\beta} \subset b \bar{\beta}$, il en résulte que la fonction $N E G$ se calcule directement à partir de la relation $\mathcal{R}$ et, de plus, que si $N E G(b) \neq \emptyset$ pour chaque $b \in B$, la restriction de $\mathcal{R}$ à $N E G(B) \times B$ est déterminée par la seule donnée de la restriction de l'ordre $\leq$ à $B$.

Pour achever la preuve, nous supposons désormais $\mathcal{R}$ telle que $N E G(b) \neq$ $\emptyset$ pour chaque $b \in B$.

Soit $T \in 2^{2^{B}}$ le plus petit treillis pour les opérations $\cup$ et $\cap \operatorname{de}\left(2^{B}, \subset\right)$ qui contienne tous les sous-ensembles $b_{0}:=[b \leq](b \in B)$ de $B$; il est classique que $(T, \subset)$ est un treillis distributif en tant que sous-treillis du treillis distributif $\left(2^{B}, \subset\right)$. En outre $b \longrightarrow b_{0}$ est un isomorphisme (d'ordre) de $B$ sur une partie $B_{0}$ de $T$.

Posons aussi $b^{0}:=B \backslash[b \leq] \subset 2^{B}$ pour chaque $b \in B$. Cet ensemble appartient à $T$ et il est l'union des $b_{0}^{\prime} \in B_{0} \backslash\left[\begin{array}{ll}b_{0} & C\end{array}\right]$, ce qui montre que $\left(b^{0}, b_{0}\right)$ est un clivage de $T$ et que la base et la cobase de ce treillis sont $B_{0}$ et $B^{0}=\left\{b^{0}: b \in B\right\}$.

D'après le lemme 8.5, la relation basique $R_{0} \subset B^{0} \times B_{0}$ de $T$ est isomorphe à la restriction $\mathcal{R}^{\prime}$ de $\mathcal{R}$ à $\bar{B}^{\prime} \times B$ où $\bar{B}^{\prime}=N E G(B) \subset \bar{B}$. 
On vérifie maintenant que $\bar{B}^{\prime}=\bar{B}$. Soit $\bar{b}$ un élément de la cobase $\bar{B}$. Si $b \in \bar{b} \beta$, on a $b^{\prime} \in \bar{b} \beta$ pour tout $b^{\prime} \in B \cap[\leq b]$ d'après la définition de $\leq$. Par conséquent, l'image de $\bar{b} \beta$ dans $2^{B}$ par l'isomorphisme $b \longrightarrow b_{0}$ est un élément du treillis $T$. On a donc $\bar{b} \in \bar{B}^{\prime}$, montrant que $\bar{B}^{\prime}=\bar{B}$, c'est-à-dire que $T$ est bien le treillis engendré par $\mathcal{R}$

Les calculs dans le treillis distributif $T$ sont facilités par l'identité de Morgan

$$
t \bar{\beta}=N E G(B \backslash t \beta) \quad(t \in T)
$$

que l'on déduit immédiatement du lemme 8.5 en identifiant, par complémentation ensembliste, le treillis antiisomorphe $\bar{T} \in 2^{2^{\bar{B}}}$ à un sous treillis de $\left(2^{B}, \subset\right)$.

Il est curieux que l'égalité numérique $\operatorname{card}(B)=\operatorname{card}(\bar{B})$ entre la base et la cobase reste vraie dans la variété des treillis modulaires dont les treillis distributifs sont une sous variété.

Pour avoir des exemples plus substantiels, nous considérons le treillis des partitions d'un entier $n$. Nous faisons référence à un article récent de C.Greene et Kleitman [G-K] dans lequel le lecteur trouvera exposés les principaux résultats sur l'ensemble des partitions en tant que treillis ( $\mathrm{cf}$. aussi S.Kim dans un article à paraître).

Nous adoptons la notation exponentielle pour les partitions, et appelons équerre une partition du type $1^{k} c$, i.e. une partition qui a au plus une part différente de 1.

Proposition 8.6. - La base du treillis des partitions d'un entier fixén est constituée des partitions, ayant trois parts différentes au plus, du type $a b^{k} c$, où $k \geq 2$ et $a<b<c$, avec les cas dégénérés suivants : $a b^{k}, b^{k} c, b^{k}$, $(k \geq 2)$, et bc.

La cobase est formée des partitions transposées des éléments de la base. L'intersection de la base et de la cobase est constituée par les équerres.

Pour que deux partitions $g, \bar{g}$, déterminent un clivage, il faut que l'une d'entre elles soit une équerre. Réciproquement, chaque équerre donne deux clivages, selon qu'elle est considérée comme un élément de la base ou de la cobase.

Preuve. - En effet, deux partitions sont consécutives dans le diagramme des partitions ssi l'on obtient le diagramme de l'une à partir de celui de l'autre en déplaçant de manière minimale une boite. Si la partition a au moins quatre parts différentes, elle ne peut être irréductible pour $\vee$. On exclut ensuite les cas $a^{h} b^{k} c^{l}$ où $h l>1$. Les cas restants se vérifient sans difficulté.

Soit maintenant un clivage $[g \leq],[\leq \bar{g}]$. La partition $g$ appartient à la base, supposons la du type $g=a b^{k} c, a<b \leq c$ où $2 \leq k$ si $b \neq c$ et 
$1 \leq k$ si $b=c$. Considérons $h=1^{n-c-1} c+1$. L'élément $g$ ne vérifie pas $g \leq h$, puisque $a<a+1$. Or $h$ est couverte par une seule partition, à savoir $h_{+}:=1^{n-c-2} 2 c$ et l'on a $g \leq h_{+}$. Donc $h$ est un élément maximal du complément de $[g \leq]$. Comme par hypothèse, ce complément est égal à $[\leq \bar{g}]$, on doit avoir $\bar{g}=h$. De plus alors $g=a c^{m}$, où $m$ est la partie entière de $n / c$ et $a$ le reste. Par conjugaison des partitions, on vérifie que symétriquement, le complément de $[h \leq]$ est un intervalle inférieur

Par exemple, les rectrices de $1^{5} 24^{2}$ sont $34^{3}, 13^{3} 5,12^{4} 6$ et $1^{7} 8$. Le lecteur pourra vérifier que le nombre des rectrices d'une partition est au plus égal à la valeur de sa deuxième plus grande part.

\section{Bibliographie}

[Bi] G. Birkoff, Lattice theory, 3ème ed., Am. Math. Soc., Providence (1967).

[Bj] A. Björner, Ordering of Coxeter groups, Contemp. Math. 34, A.M.S. (1984) 175-195.

[B-W] A. Björner, M. Wachs, Generalized quotients in Coxeter groups, Trans. A.M.S. 308 (1988) 1-37.

[Bo] N. Bourbaki, Groupes et Algèbres de Lie, Fasc 34, Hermann, Paris (1968).

[De] V.V. Deodhar, Some characterizations of Bruhat ordering on a Coxeter group, Invent. Math. 39 (1977) 187-198.

[Ch] I. Cherednik, A unification of Knizhnik-Zamolodchikov and Dunkle operators via affine Hecke algebras, Invent. Math. 106 (1991) 411-431.

[Eh] C. Ehresmann, Sur la topologie de certains espaces homogènes, Ann. Math. 35 (1934) 396-443.

[Er] H. Eriksson, Computational and combinatorial aspects of Coxeter groups, thèse, KTH Stockholm (1994).

[Fo] Fokko du Cloux, A transducer approach to Coxeter groups, prépublication (1995).

[Fu] W. Fulton, Flags, Schubert polynomials, degeneraci loci Duke Math. 65 (1992) 381-420.

[G-Ki] M. Geck, S. Kim, On the Bruhat order of finite Coxeter groups, prépublication (1996).

[G-K] C. Greene, D. Kleitman, Longest chains in the lattice of integer partitions ordered by majorization, Europ. J. Comb. 7 (1986) 1-10.

[G-R] G. Guilbaud, P. Rosenstiehl, Analyse algébrique d'un scrutin, in Ordres totaux finis, Gauthiers-Villars, Paris (1971).

[Lak] V. Lakshmibai, Geometry of $G / P$. The symplectic group and the involution $\sigma$, J. Alg. 108 (1987) 403-434.

[L-S 1] A. Lascoux, M.P. Schützenberger, Structure de Hopf de l'anneau 
de cohomologie de la variété de drapeaux, C.R. Acad. Sc. Paris 295 (1982) 629-633.

[L-S 2] A. Lascoux, M.P. Schützenberger, Symmetrization operators on polynomial rings, Funk. Anal. 21 (1987) 77-78.

[Mo] A. Molev, On Stirling partitions of the symmetric group, prépublication, Australian National University (1995).

[MRR] W.H. Mills, D.P. Robbins, H. Rumsey, Alternating sign matrices and descending plane partitions, J. Comb. Th. A (1983) 340-359.

[Pr] R.A. Proctor, Classical Bruhat orders and lexicographic shellability, J. Alg. 77 (1982) 104-126.

[Vr] D.N Verma, Möbius inversion for the Bruhat ordering on a Weyl group, Ann. Sc. Ec. Norm. Sup 4 (1971) 393-398. 
Rectrices des permutations de S

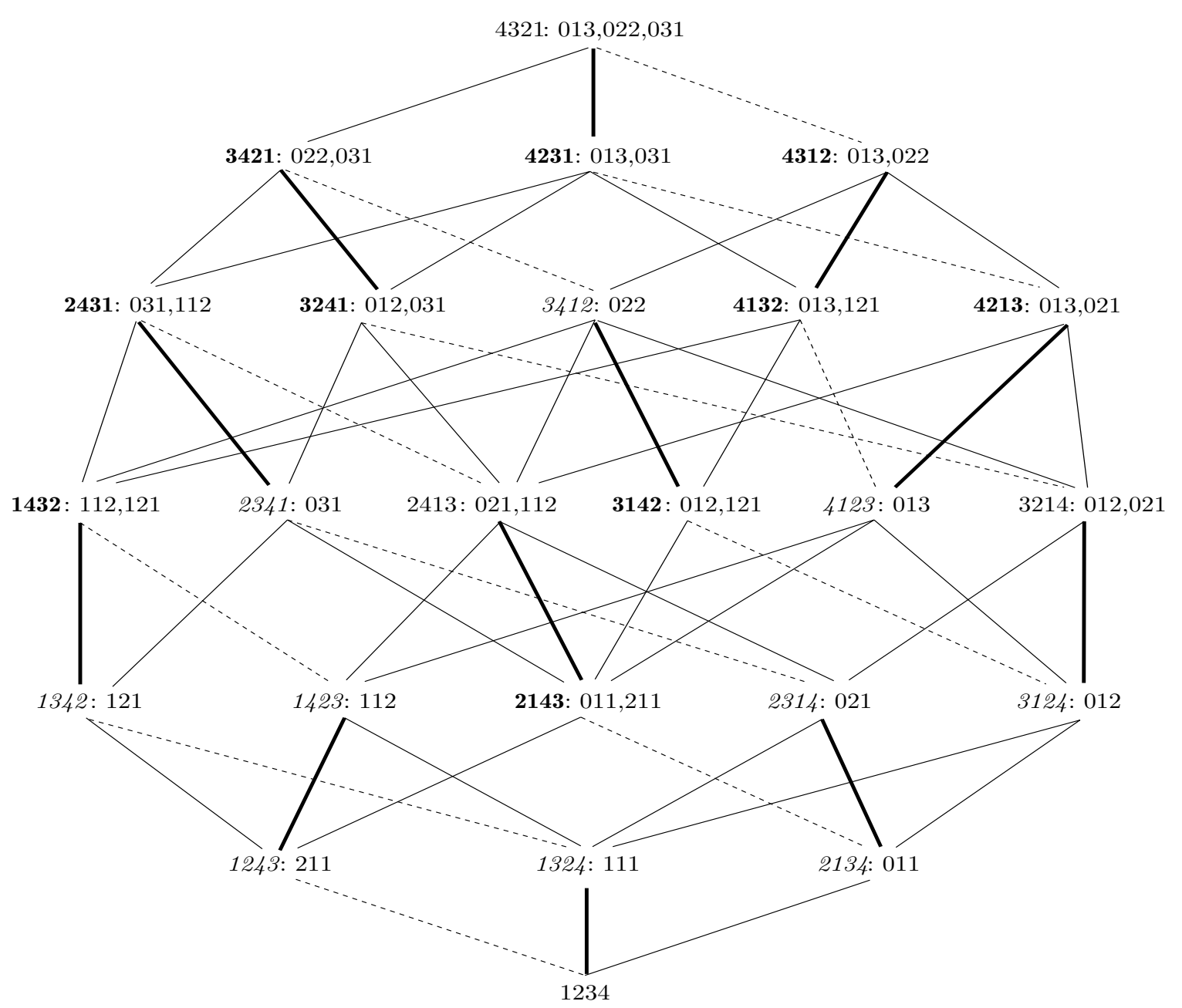

bigrassmanniennes en italique cobigrassmannienne en gras une rectrice $\left[\mathrm{r}_{0}, \mathrm{r}_{2},{ }_{3} \mathrm{r}\right]$

se note $r_{0} \mathrm{r}_{2}$ 


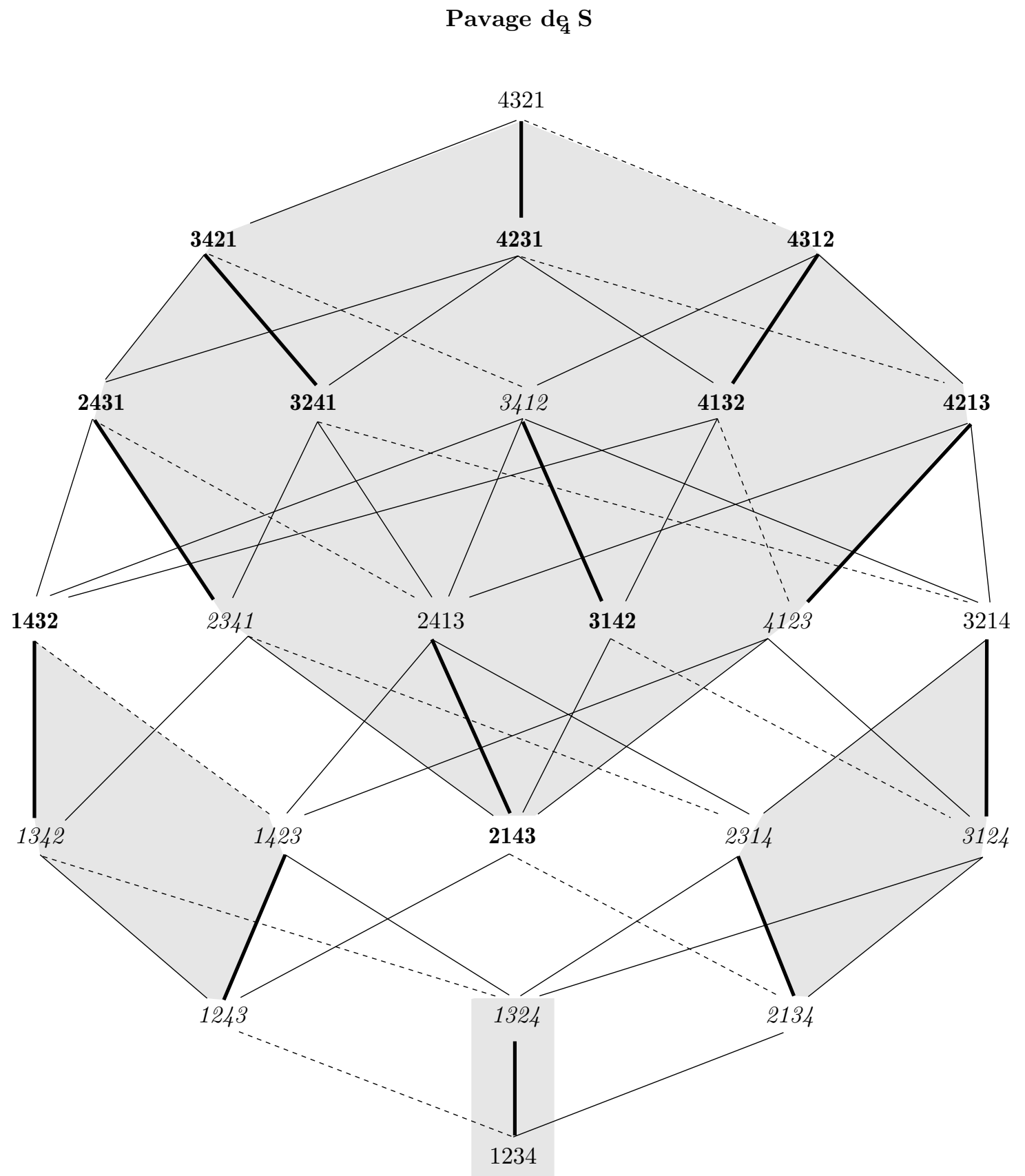

